\title{
Design, synthesis, and antibacterial activity of novel myricetin derivatives containing sulfonate
}

\author{
Shijun Su${ }^{1} \cdot$ Qing Zhou ${ }^{1} \cdot$ Xuemei Tang $^{1} \cdot$ Feng Peng ${ }^{1} \cdot$ Tingting Liu ${ }^{1}$ Liwei Liu ${ }^{1}$ Chengwei Xie ${ }^{1} \cdot$ Ming He $^{1}$. \\ Wei Xue ${ }^{1}$ (1)
}

Received: 5 December 2020 / Accepted: 12 January 2021 / Published online: 5 March 2021

(c) The Author(s) 2021

\begin{abstract}
A series of myricetin derivatives containing sulfonate groups were designed and synthesized. Preliminary antibacterial activity showed that most of the target compounds exhibited significant biological activities against Xanthomonas axonopodis pv. Citri (Xac), Ralstonia solanacearum (Rs), and Xanthomonas oryzae pv. Oryzae (Xoo). In particular, the $\mathrm{EC}_{50}$ value of compound $3 \mathrm{e}$ was $13.76 \mu \mathrm{g} / \mathrm{cm}^{3}$ against $X a c$, which was better than commercial reagents bismerthiazol $\left(50.32 \mu \mathrm{g} / \mathrm{cm}^{3}\right)$ and thiodiazole copper. $\left(83.27 \mu \mathrm{g} / \mathrm{cm}^{3}\right.$ ), and the $\mathrm{EC}_{50}$ value of compound $\mathbf{3 j}$ was $11.92 \mu \mathrm{g} / \mathrm{cm}^{3}$ against Xoo in vitro, The result was better than that of bismerthiazol $\left(72.08 \mu \mathrm{g} / \mathrm{cm}^{3}\right)$ and thiodiazole copper $\left(99.26 \mu \mathrm{g} / \mathrm{cm}^{3}\right)$. Compound $\mathbf{3 j}$ displayed the better in vivo activity against rice bacterial leaf blight than bismerthiazol and thiodiazole copper. Meanwhile, the antibacterial mechanism of compounds $\mathbf{3 e}$ and $\mathbf{3 j}$ was studied by scanning electron microscope (SEM). These results suggested that myricetin derivatives containing sulfonate can be considered as a new antibacterial reagents.
\end{abstract}

\section{Graphic abstract}
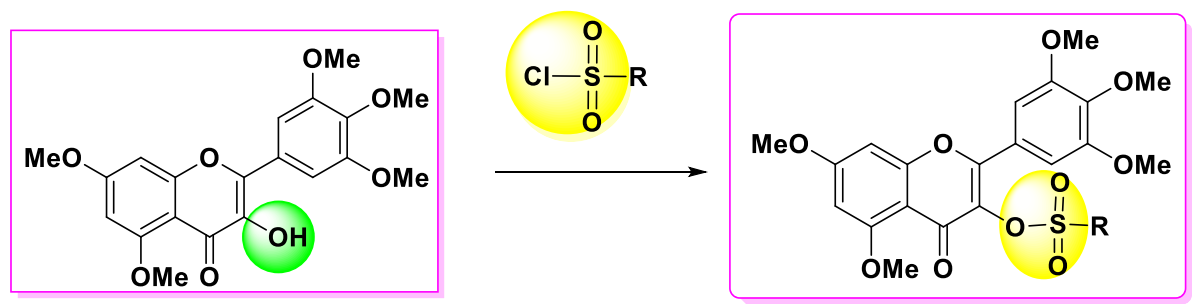

Keywords Myricetin derivatives $\cdot$ Sulfonate $\cdot$ Antibacterial activities $\cdot$ Crystal structure $\cdot$ Scanning electron microscopy

Wei Xue

wxue@gzu.edu.cn

1 Key Laboratory of Green Pesticide and Agricultural Bioengineering, Ministry of Education, Research and Development Center for Fine Chemicals, Guizhou University, Guiyang 550025, People's Republic of China 


\section{Introduction}

Plant bacterial diseases are frequently encountered in agricultural production and difficult to manage, such as citrus canker, tobacco bacterial wilt and rice bacterial leaf blight, which can be caused by Xanthomonas axonopodis pv. Citri (Xac), Ralstonia solanacearum (Rs), and Xanthomonas oryzae pv. oryzae (Xoo), respectively. Meanwhile, these bacterial diseases have a serious impact on the yield of crops every year [1-3]. Although there are many traditional agricultural fungicides on the market at present, the extensive application and abuse of antibacterial drugs have not only increased the resistance of pathogenic genes, but also caused serious environmental pollution and affect human health [4-6]. Therefore, it is of great significance to develop a new kind of antibacterial reagents with high efficiency and novel mechanism.

Myricetin, also named as 3',4',5',3,5,7-hexahydroxyflavonol (Fig. 1A), is a class of flavonoids widely found in fruits, vegetables, teas, and so on [7]. Myricetin has been disclosed to display extensive biological activities, such as antibacterial [8, 9], antiviral [10, 11], antioxidant [12, 13], anti-inflammatory $[14,15]$, anticancer $[16,17]$, hypoglycemic [18] activities, and cardioprotective effects [19]. Due to wide range of plant sources and biological activity, myricetin and its derivatives have attracted more and more attention of researchers in recent years. In our previous study, we have demonstrated that myricetin derivatives exhibited excellent antibacterial activity [20, 21] (Fig. 1B and C). Myricetin derivatives could destroy the integrity of the cell membrane and cause the death of bacteria, thus achieving the objective of bacteriostasis [22].

Sulfonate derivatives, owing to physicochemical properties, have a strong affinity for lipid phases and can cross the cuticle membrane easily to bind to target sites [23], and compounds containing aryl sulfonate moieties have received considerable attention due to extensive biological activities, and it has been proven to possess antiviral [24, 25], antibacterial [26-28], insecticidal [29, 30], anticancer [31, 32], and other biological activity [33]. They were widely used in agricultural industries, medicine researches and other fields. We theorized that introducing sulfonate groups into myricetin might generate novel lead molecules with better physicochemical properties. Therefore, a series of novel myricetin derivatives containing sulfonate were designed (Fig. 2), synthesized (Scheme 1), and their in vitro and in vivo antibacterial activity was evaluated. To the best of our knowledge, this is the first report on the synthesis and

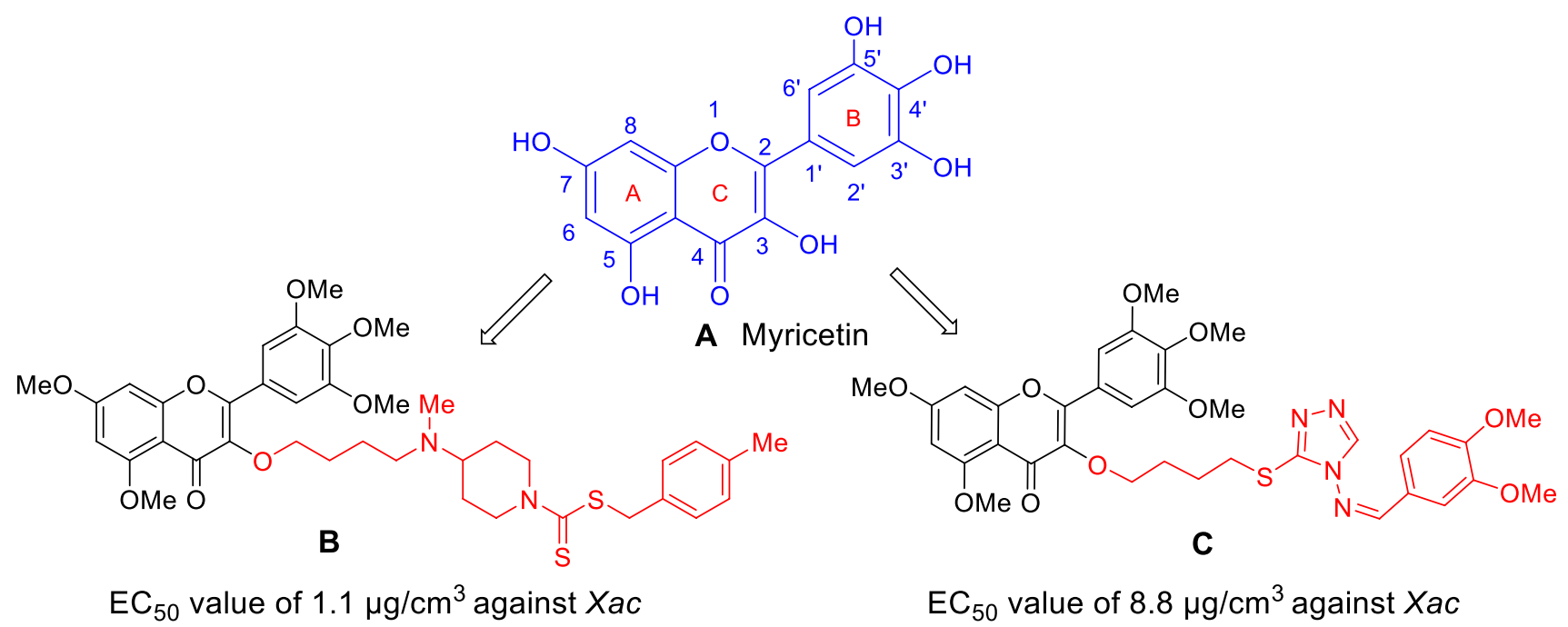

Fig. 1 Structure of myricetin and its derivatives with antibacterial activity

Fig. 2 Design strategy of the title compounds
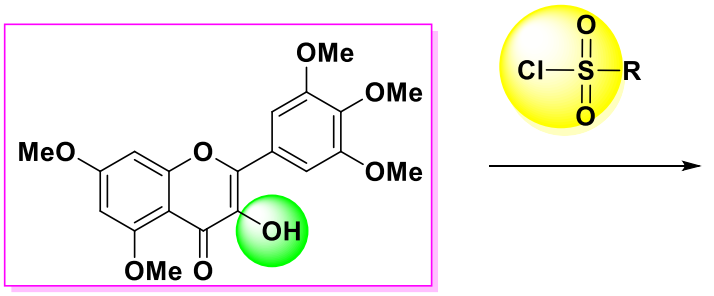
Scheme 1<smiles>CC(=O)O[C@H](C)C(=O)Oc1c(-c2cc(O)c(O)c(O)c2)oc2cc(O)cc(O)c2c1=O</smiles><smiles>COc1cc(OC)c2c(=O)c(OC(O)C(O)CO)c(-c3cc(OC)c(OC)c(OC)c3)oc2c1</smiles><smiles>[R]OS(=O)(=O)Oc1c(-c2cc(OC)c(OC)c(OC)c2)oc2cc(OC)cc(OC)c2c1=O</smiles>

3 3a: $\mathrm{R}=\mathrm{Ph}$

3d: $\mathrm{R}=2-\mathrm{NO}_{2}-\mathrm{Ph}$

3g: $\mathrm{R}=4-\mathrm{F}-\mathrm{Ph}$

3j: $\mathrm{R}=3-\mathrm{Cl}-\mathrm{Ph}$ $3 \mathrm{~m}: \mathrm{R}=3-\mathrm{Br}-\mathrm{Ph}$

3p: $\mathrm{R}=4-\mathrm{CF}_{3} \mathrm{O}-\mathrm{Ph}$

3s: $\mathrm{R}=2$-Thienyl

3v: $\mathrm{R}=4$-Acetamido-Ph 3b: $\mathrm{R}=4-\mathrm{CH}_{3}-\mathrm{Ph}$
3e: $\mathrm{R}=4-\mathrm{NO}_{2}-\mathrm{Ph}$
3h: $\mathrm{R}=3-\mathrm{F}-\mathrm{Ph}$
3k: $\mathrm{R}=4-\mathrm{Cl}-\mathrm{Ph}$
3n: $\mathrm{R}=4-\mathrm{Br}-\mathrm{Ph}$
3q: $\mathrm{R}=4-$ tert-Butyl-Ph
3t: $\mathrm{R}=3-\mathrm{Pyridyl}$

3c: $\mathrm{R}=4-\mathrm{CH}_{3} \mathrm{O}-\mathrm{Ph}$

3f: $\mathrm{R}=2-\mathrm{F}-\mathrm{Ph}$

3i: $\mathrm{R}=2-\mathrm{Cl}-\mathrm{Ph}$

3I: $\mathrm{R}=2-\mathrm{Br}-\mathrm{Ph}$

30: $\mathrm{R}=3-\mathrm{CF}_{3}-\mathrm{Ph}$

3r: $R=4-C N-P h$

3u: $R=8-$-Quinolinyl antibacterial activity evaluation of myricetin derivatives containing sulfonate moiety.

\section{Results and discussion}

The synthetic route of myricetin derivatives containing sulfonate is shown in Scheme 1. Myricetrin, methyl iodide, and potassium carbonate were mixed and stirred at room temperature to give intermediate 1 and then continued to react with concentrated hydrochloric acid to get intermediate 2. Intermediate $\mathbf{2}$ was treated with different benzenesulfonyl chlorides in the presence of potassium carbonate to obtain the target products $\mathbf{3 a}-\mathbf{3 v}$ in good yields ranging from 53 to $89 \%$.

The structures of all compounds were confirmed by ${ }^{1} \mathrm{H}$ NMR, ${ }^{13} \mathrm{C}$ NMR, ${ }^{19} \mathrm{~F}$ NMR, and HRMS. The representative data of compound $3 \mathrm{~b}$ are shown below. In the ${ }^{1} \mathrm{H}$ NMR spectrum, the multiple signal $\delta=6.57-7.57 \mathrm{ppm}$ demonstrated the presence of aromatic ring protons, the singlet signal of 3.6-4.0 ppm belonged to $-\mathrm{OCH}_{3}$ on myricetin, and the singlet signal of $2.34 \mathrm{ppm}$ indicates the presence of $-\mathrm{CH}_{3}$ group. Typical chemical shifts at $\delta=145.45$, 133.91, and $21.56 \mathrm{ppm}$ in ${ }^{13} \mathrm{C}$ NMR spectrum confirmed the existence of $-\mathrm{O}-\mathrm{C}-,-\mathrm{S}-\mathrm{C}-$, and $-\mathrm{CH}_{3}$ groups, respectively. In ${ }^{19} \mathrm{~F}$ NMR spectrum, the obvious singlets at -109 . $88,-107.54,-103.47,-61.51$, and -56.74 ppm confirm,
Table 1 Crystal data of title compound 3n

\begin{tabular}{ll}
\hline Crystal data & \\
$\mathrm{C}_{26} \mathrm{H}_{23} \mathrm{BrO}_{10} \mathrm{~S}$ & $T=293 \mathrm{~K}$ \\
$\mathrm{FW}=607.41$ & $\alpha=90^{\circ}$ \\
Monoclinic /C12/c 1 & $\beta=107.9680(10)^{\circ}$ \\
$a=18.2079(6) \AA$ & $\gamma=90^{\circ}$ \\
$b=20.8420(6) \AA$ & $V=5117.0(3) \AA^{3}$ \\
$c=14.1753(4) \AA$ & $Z=8$ \\
$F(000)=2480$ & $\rho=1.577 \mathrm{mg} / \mathrm{m}^{3}$ \\
Data collection & \\
$T_{\text {min }}=0.8914, T_{\max }=0.9866$ & Absorption coefficient: $3.465 \mathrm{~mm}^{-1}$ \\
18,533 Reflections collected & $-21<=\mathrm{h}<=21,-24<=\mathrm{k}<=24,-$ \\
& $13<=1<=16$ \\
4341 independent reflections & $\theta_{\text {min }}=3.318^{\circ}, \theta_{\max }=65.115^{\circ}$ \\
Refinement & $R$ indices $($ all data $) R_{1}=0.0548, \mathrm{w}_{2}=0.1447$ \\
349 parameters & $R$ indices $[I>2 \sigma(I)] R_{1}=0.0536, \mathrm{w}_{2}=0.1440$ \\
Goodness-of-fit: 1.052 & $\Delta \rho_{\text {max }}=0.818 \mathrm{e} \AA^{-3}, \Delta \rho_{\min }=-0.779 \AA^{-3}$ \\
Extinction coefficient $=0.00125(12)$ &
\end{tabular}


Fig. 3 Crystal structure and crystal packing diagram of compound 3n
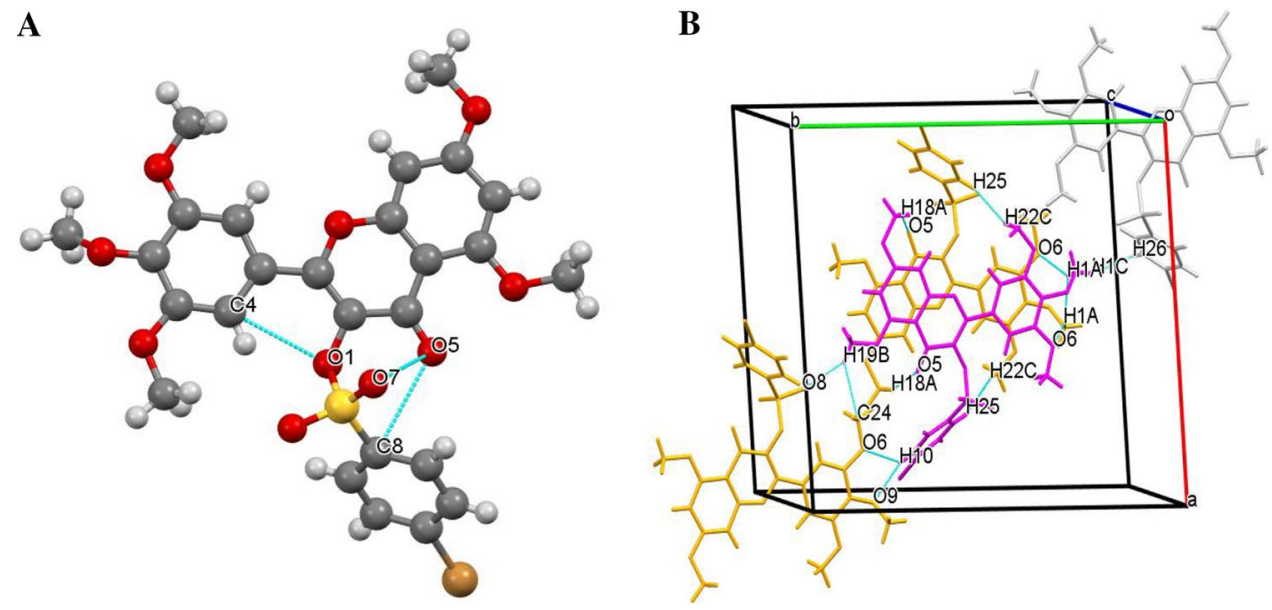

respectively, the presence of a 3-F atom, a 2-F atom, a 4-F atom, a $3-\mathrm{CF}_{3}$, and a $4-\mathrm{OCF}_{3}$ group at the benzene ring. The strong presence of $[\mathrm{M}+\mathrm{H}]^{+}$ions indicates the stable state of the title compounds.

To further confirm the structure of synthesized compounds, the molecular structure of $\mathbf{3 n}$ was studied as a representative example by single-crystal $X$-ray analysis. The tested single crystal was crystallized from the mixture of acetone and $\mathrm{N}, \mathrm{N}$-dimethylformamide solution under room temperature. The crystal diffraction data are presented in Table 1. Crystal structure diagram and crystal packing diagram are shown in Fig. $3 \mathrm{~A}$ and $\mathrm{B}$, respectively. Figure $3 \mathrm{~A}$ shows that the intramolecular hydrogen bond $\mathrm{C}_{4} \cdots \mathrm{O}_{1}, \mathrm{O}_{7} \cdots \mathrm{O}_{5}, \mathrm{O}_{5} \cdots \mathrm{C}_{8}$ in crystal combines with the skeleton of myricetin and sulfonate. As showed in Fig. 3B, the four intermolecular hydrogen bonds $\mathrm{H}_{25} \cdots \mathrm{H}_{22}-\mathrm{C}, \mathrm{H}_{18} \mathrm{~A} \cdots \mathrm{O} 5, \mathrm{C}-\mathrm{H}_{1} \mathrm{~A} \cdots \mathrm{O}_{6}$, and $\mathrm{C}-\mathrm{H}_{1} \mathrm{~A} \cdots \mathrm{H}_{1} \mathrm{~A}-\mathrm{C}$ constructed the three-dimensional structure of target compound 3n. The deposition number is CCDC-2017276.

\section{Antibacterial activity}

The in vitro antibacterial activities of the title compounds 3a-3v against three phytopathogenic bacteria ( $\mathrm{Xac}, \mathrm{Rs}$, and Xoo) were tested by turbidimeter [34, 35]. The commercial bactericides bismerthiazol (BT) and thiadiazole-copper (TC) were used as a positive control under the same conditions, the bioassay results showed (Table 2) that some of the compounds exhibited considerable antibacterial effects against Xac, Rs, and Xoo. Especially, compounds $\mathbf{3 e}, \mathbf{3 i}$, 3k, 3n, 3o, and 3q showed excellent antibacterial activity against $X a c$ at $100 \mu \mathrm{g} / \mathrm{cm}^{3}$, with the inhibition rates of 95 , $82,88,93,90$, and $82 \%$, respectively, which were superior to the commercial bactericides BT $(66 \%)$ and TC (56\%). Meanwhile, the title compounds $\mathbf{3 d}, \mathbf{3 h}, \mathbf{3 j}, \mathbf{3 m}$, and $\mathbf{3 p}$ exhibited obvious antibacterial activity against $R s$ at $100 \mu \mathrm{g} /$ $\mathrm{cm}^{3}$, with the inhibition rates of $86,87,92,90$, and $87 \%$, respectively, which exceeded both $\mathrm{BT}(70 \%)$ and TC (57\%). And the antibacterial activity of the compounds $\mathbf{3 b}, \mathbf{3} \mathbf{c}, \mathbf{3 h}$, $\mathbf{3 j}$, and $\mathbf{3 r}$ against $X o o$ at $100 \mu \mathrm{g} / \mathrm{cm}^{3}$ were $90 \%, 89 \%, 91 \%$, $94 \%, 80 \%$, respectively, which were better than BT $(50 \%)$ and TC (47\%). Obviously, most of the myricetin derivatives containing sulfonate had better inhibitory effects against bacteria than lead compound myricetin.

In order to further confirm the antibacterial activities of title compounds, their $\mathrm{EC}_{50}$ values were determined. The results indicated that compounds $\mathbf{3 e}, \mathbf{3 i}, \mathbf{3 k}, \mathbf{3 n}$, and $\mathbf{3 o}$ exhibited prominent antibacterial activity against $\mathrm{Xac}$ with $\mathrm{EC}_{50}$ values of $13.72,23.19,19.81,16.89$, and $19.19 \mu \mathrm{g} / \mathrm{cm}^{3}$ (Table 3), which were superior to BT $\left(50.32 \mu \mathrm{g} / \mathrm{cm}^{3}\right)$ and TC $\left(83.27 \mu \mathrm{g} / \mathrm{cm}^{3}\right)$. Meanwhile, Table 3 indicated that compounds $\mathbf{3 a}, \mathbf{3 d}, \mathbf{3 h}$, and $\mathbf{3 j}$ displayed excellent antibacterial activity against $R s$ with the $\mathrm{EC}_{50}$ values of 26.66, 25.07, 21.64 , and $17.11 \mu \mathrm{g} / \mathrm{cm}^{3}$, respectively, which were better than BT $\left(43.53 \mu \mathrm{g} / \mathrm{cm}^{3}\right)$ and TC $\left(64.32 \mu \mathrm{g} / \mathrm{cm}^{3}\right)$. Compounds $\mathbf{3 b}, \mathbf{3 c}, \mathbf{3 h}$, and $\mathbf{3 j}$ have fine antibacterial activity against Xoo with $\mathrm{EC}_{50}$ values of $21.93,24.98,19.53$, and $11.92 \mu \mathrm{g} /$ $\mathrm{cm}^{3}$, respectively, which were better than BT $\left(72.08 \mu \mathrm{g} / \mathrm{cm}^{3}\right)$ and TC $\left(99.26 \mu \mathrm{g} / \mathrm{cm}^{3}\right)$. The results showed that these compounds could be further studied as potential compounds in search of novel antibacterial agents.

\section{Structure-activity relationships (SAR) of antibacterial activities}

It can be seen from Tables 2 and 3 that the substituents greatly impact the inhibition effects against bacteria. When the $\mathrm{R}$ group were $4-\mathrm{NO}_{2}-\mathrm{C}_{6} \mathrm{H}_{5}(\mathbf{3 e}), 4-\mathrm{Cl}-\mathrm{C}_{6} \mathrm{H}_{5}$ (3k), 4- $\mathrm{Br}-\mathrm{C}_{6} \mathrm{H}_{5}(\mathbf{3 n})$, and $3-\mathrm{CF}_{3}-\mathrm{C}_{6} \mathrm{H}_{5}(\mathbf{3 o})$, their inhibition rates against $\mathrm{Xac}$ at $100 \mu \mathrm{g} / \mathrm{cm}^{3}$ were $95,88,93$, and 
Table 2 Inhibition rate of title compounds $\mathbf{3 a}-\mathbf{3 v}$ against $\mathrm{Xac}$, $R s$, and $X o o$ in vitro

\begin{tabular}{|c|c|c|c|c|c|c|c|}
\hline \multirow[t]{2}{*}{ Compd } & \multirow[t]{2}{*}{$\mathrm{R}$} & \multicolumn{2}{|l|}{$\mathrm{Xac} / \%$} & \multicolumn{2}{|l|}{$R s / \%$} & \multicolumn{2}{|l|}{ Xoo/\% } \\
\hline & & $100 \mu \mathrm{g} / \mathrm{cm}^{3}$ & $50 \mu \mathrm{g} / \mathrm{cm}^{3}$ & $100 \mu \mathrm{g} / \mathrm{cm}^{3}$ & $50 \mu \mathrm{g} / \mathrm{cm}^{3}$ & $100 \mu \mathrm{g} / \mathrm{cm}^{3}$ & $50 \mu \mathrm{g} / \mathrm{cm}^{3}$ \\
\hline $3 \mathbf{a}$ & $\mathrm{C}_{6} \mathrm{H}_{5}$ & 48 & 27 & 85 & 54 & 48 & 30 \\
\hline $3 \mathbf{b}$ & $4-\mathrm{CH}_{3}-\mathrm{C}_{6} \mathrm{H}_{5}$ & 50 & 28 & 58 & 27 & 90 & 70 \\
\hline $3 \mathbf{c}$ & $4-\mathrm{CH}_{3} \mathrm{O}-\mathrm{C}_{6} \mathrm{H}_{5}$ & 41 & 21 & 34 & 12 & 89 & 75 \\
\hline 3d & $2-\mathrm{NO}_{2}-\mathrm{C}_{6} \mathrm{H}_{5}$ & 79 & 59 & 86 & 56 & 54 & 30 \\
\hline $3 e$ & $4-\mathrm{NO}_{2}-\mathrm{C}_{6} \mathrm{H}_{5}$ & 95 & 78 & 76 & 39 & 60 & 40 \\
\hline 3f & $2-\mathrm{F}-\mathrm{C}_{6} \mathrm{H}_{5}$ & 60 & 40 & 70 & 43 & 56 & 34 \\
\hline $3 g$ & $4-\mathrm{F}-\mathrm{C}_{6} \mathrm{H}_{5}$ & 43 & 27 & 71 & 43 & 37 & 17 \\
\hline $3 \mathbf{h}$ & $3-\mathrm{F}-\mathrm{C}_{6} \mathrm{H}_{5}$ & 71 & 44 & 87 & 51 & 91 & 69 \\
\hline $3 \mathbf{i}$ & $2-\mathrm{Cl}-\mathrm{C}_{6} \mathrm{H}_{5}$ & 82 & 41 & 75 & 32 & 73 & 48 \\
\hline $\mathbf{3 j}$ & $3-\mathrm{Cl}-\mathrm{C}_{6} \mathrm{H}_{5}$ & 60 & 46 & 92 & 66 & 94 & 72 \\
\hline $3 \mathbf{k}$ & $4-\mathrm{Cl}-\mathrm{C}_{6} \mathrm{H}_{5}$ & 88 & 68 & 57 & 35 & 69 & 34 \\
\hline 31 & $2-\mathrm{Br}-\mathrm{C}_{6} \mathrm{H}_{5}$ & 75 & 49 & 65 & 37 & 33 & 17 \\
\hline $3 \mathrm{~m}$ & $3-\mathrm{Br}-\mathrm{C}_{6} \mathrm{H}_{5}$ & 63 & 34 & 90 & 60 & 80 & 50 \\
\hline $3 n$ & $4-\mathrm{Br}-\mathrm{C}_{6} \mathrm{H}_{5}$ & 93 & 72 & 67 & 41 & 55 & 29 \\
\hline 30 & $3-\mathrm{CF}_{3}-\mathrm{C}_{6} \mathrm{H}_{5}$ & 90 & 69 & 71 & 55 & 74 & 36 \\
\hline $3 \mathbf{p}$ & $4-\mathrm{CF}_{3} \mathrm{O}-\mathrm{C}_{6} \mathrm{H}_{5}$ & 38 & 22 & 87 & 63 & 31 & 22 \\
\hline $3 \mathbf{q}$ & 4-tert-Butyl- $\mathrm{C}_{6} \mathrm{H}_{5}$ & 82 & 54 & 64 & 53 & 67 & 46 \\
\hline $3 \mathbf{r}$ & $4-\mathrm{CN}-\mathrm{C}_{6} \mathrm{H}_{5}$ & 64 & 55 & 55 & 38 & 78 & 57 \\
\hline $3 s$ & 2-Thienyl & 45 & 29 & 36 & 14 & 35 & 24 \\
\hline $3 t$ & 3-Pyridyl & 55 & 37 & 54 & 35 & 36 & 19 \\
\hline $3 \mathbf{u}$ & 3-Quinolinyl & 38 & 21 & 40 & 16 & 23 & 13 \\
\hline $3 \mathbf{v}$ & 4-Acetamido- $\mathrm{C}_{6} \mathrm{H}_{5}$ & 26 & 17 & 63 & 31 & 40 & 20 \\
\hline Myr & - & 47 & 36 & 65 & 40 & 40 & 27 \\
\hline BT & - & 66 & 42 & 70 & 44 & 50 & 31 \\
\hline $\mathrm{TC}$ & - & 56 & 38 & 57 & 36 & 47 & 29 \\
\hline
\end{tabular}

Table $3 \mathrm{EC}_{50}$ values of some target compounds against Xac, Хoo, and $R s$

\begin{tabular}{|c|c|c|c|c|}
\hline \multirow[t]{2}{*}{ Compound } & \multirow[t]{2}{*}{$\mathrm{R}$} & \multicolumn{3}{|c|}{$\mathrm{EC}_{50} / \mu \mathrm{g} \mathrm{cm}^{-3}$} \\
\hline & & $X a c$ & Хоо & $R s$ \\
\hline $3 \mathbf{a}$ & $\mathrm{C}_{6} \mathrm{H}_{5}$ & - & - & 26.66 \\
\hline $3 \mathbf{b}$ & 4- $\mathrm{CH}_{3}-\mathrm{C}_{6} \mathrm{H}_{5}$ & - & 21.93 & - \\
\hline $3 c$ & $4-\mathrm{CH}_{3} \mathrm{O}-\mathrm{C}_{6} \mathrm{H}_{5}$ & - & 24.98 & - \\
\hline 3d & $2-\mathrm{NO}_{2}-\mathrm{C}_{6} \mathrm{H}_{5}$ & - & - & 25.07 \\
\hline $3 e$ & $4-\mathrm{NO}_{2}-\mathrm{C}_{6} \mathrm{H}_{5}$ & 13.76 & - & - \\
\hline $3 h$ & $3-\mathrm{F}-\mathrm{C}_{6} \mathrm{H}_{5}$ & & 19.53 & 21.64 \\
\hline $3 \mathbf{i}$ & $2-\mathrm{Cl}-\mathrm{C}_{6} \mathrm{H}_{5}$ & 23.19 & - & - \\
\hline $3 \mathbf{j}$ & $3-\mathrm{Cl}-\mathrm{C}_{6} \mathrm{H}_{5}$ & - & 11.92 & 17.11 \\
\hline $3 \mathbf{k}$ & $4-\mathrm{Cl}-\mathrm{C}_{6} \mathrm{H}_{5}$ & 19.81 & - & - \\
\hline $3 \mathrm{~m}$ & $3-\mathrm{Br}-\mathrm{C}_{6} \mathrm{H}_{5}$ & - & 35.98 & 35.04 \\
\hline $3 n$ & $4-\mathrm{Br}-\mathrm{C}_{6} \mathrm{H}_{5}$ & 16.89 & - & - \\
\hline 30 & $3-\mathrm{CF}_{3}-\mathrm{C}_{6} \mathrm{H}_{5}$ & 19.19 & - & - \\
\hline $3 \mathbf{p}$ & $4-\mathrm{CF}_{3} \mathrm{O}-\mathrm{C}_{6} \mathrm{H}_{5}$ & - & - & 33.96 \\
\hline $3 q$ & 4-tert-Butyl- $\mathrm{C}_{6} \mathrm{H}_{5}$ & 29.67 & - & - \\
\hline $3 \mathbf{r}$ & $4-\mathrm{CN}-\mathrm{C}_{6} \mathrm{H}_{5}$ & - & 29.47 & - \\
\hline Myr & - & 92.34 & 108.43 & 68.79 \\
\hline BT & - & 50.32 & 72.08 & 43.53 \\
\hline TC & - & 83.27 & 99.26 & 64.32 \\
\hline
\end{tabular}

$90 \%$, respectively, which were superior to the BT (66\%), TC (56\%), myricitin (47\%), 3a $\left(\mathrm{R}=\mathrm{C}_{6} \mathrm{H}_{5}, 48 \%\right), 3 \mathrm{~b}\left(\mathrm{R}=4-\mathrm{CH}_{3}-\right.$ $\left.\mathrm{C}_{6} \mathrm{H}_{5}, 50 \%\right)$, and $3 \mathbf{c}\left(\mathrm{R}=4-\mathrm{OCH}_{3}-\mathrm{C}_{6} \mathrm{H}_{5}, 41 \%\right)$. The results indicated that the electron-absorbing groups on aromatic rings were favorable for the antibacterial activity against Xac. The designated compounds $3 \mathbf{d}\left(\mathrm{R}=2-\mathrm{NO}_{2}-\mathrm{C}_{6} \mathrm{H}_{5}\right), \mathbf{3 h}$ $\left(\mathrm{R}=3-\mathrm{F}-\mathrm{C}_{6} \mathrm{H}_{5}\right), 3 \mathbf{j}\left(\mathrm{R}=3-\mathrm{Cl}-\mathrm{C}_{6} \mathrm{H}_{5}\right), 3 \mathbf{m}\left(\mathrm{R}=3-\mathrm{Br}_{-} \mathrm{C}_{6} \mathrm{H}_{5}\right)$, and $3 \mathbf{p}\left(\mathrm{R}=4-\mathrm{OCF}_{3}-\mathrm{C}_{6} \mathrm{H}_{5}\right)$ also effectively enhance the antibacterial activity against $R s$ at $100 \mu \mathrm{g} / \mathrm{cm}^{3}$, with inhibition rates of $86,87,92,90$, and $87 \%$, respectively, which exceeded BT $(70 \%)$ and TC $(57 \%)$, myricitin $(65 \%), 3 \mathbf{b}$ $\left(\mathrm{R}=4-\mathrm{CH}_{3}-\mathrm{C}_{6} \mathrm{H}_{5}, 58 \%\right)$, and $3 \mathrm{c}\left(\mathrm{R}=4-\mathrm{OCH}_{3}-\mathrm{C}_{6} \mathrm{H}_{5}, 34 \%\right)$ thus, the electron-absorbing groups on aromatic rings were favorable for the antibacterial activity against $R s$, the electron-donating groups on the aromatic rings were not favorable the biological activity of the corresponding compounds against $R s$. At the same time, when the $\mathrm{R}$ group were 4- $\mathrm{CH}_{3}-$ $\mathrm{C}_{6} \mathrm{H}_{5}(\mathbf{3 b}), 4-\mathrm{OCH}_{3}-\mathrm{C}_{6} \mathrm{H}_{5}(\mathbf{3 c}), 3-\mathrm{F}-\mathrm{C}_{6} \mathrm{H}_{5}(\mathbf{3 h}), 3-\mathrm{Cl}-\mathrm{C}_{6} \mathrm{H}_{5}$ (3j), and $3-\mathrm{Br}_{-} \mathrm{C}_{6} \mathrm{H}_{5}(\mathbf{3 r})$, corresponding compounds possess better antibacterial activity against $\mathrm{Xoo}$, with inhibition rates of $90,89,91,94$, and $80 \%$, respectively, which were better than that of BT $(50 \%)$, TC $(47 \%)$, myricitin (40\%), and $3 \mathbf{a}\left(\mathrm{R}=\mathrm{C}_{6} \mathrm{H}_{5}, 48 \%\right)$, the results showed that 
Table 4 Curative and protection effects of compound $\mathbf{3 j}$ against Xoo under greenhouse conditions at $200 \mu \mathrm{g} / \mathrm{cm}^{3}$

\begin{tabular}{llllll}
\hline Treatment & \multicolumn{2}{l}{ Curative activity } & & \multicolumn{2}{l}{ Protection activity } \\
\cline { 2 - 3 } \cline { 5 - 6 } & $\begin{array}{l}\text { Disease } \\
\text { index } / \%\end{array}$ & $\begin{array}{l}\text { Control } \\
\text { efficiency } \\
1 \%\end{array}$ & & $\begin{array}{l}\text { Disease } \\
\text { index } / \%\end{array}$ & $\begin{array}{l}\text { Control } \\
\text { efficiency } \\
1 \%\end{array}$ \\
\hline $\mathbf{3 j}$ & 50 & 40 & & 44 & 47 \\
Bismerthiazol & 55 & 35 & & 51 & 39 \\
Thiodiazole-copper & 59 & 30 & & 61 & 27 \\
Negative control & 85 & - & 85 & - \\
\hline
\end{tabular}

electron-withdrawing groups on 3-position of aromatic rings and electron-donating groups on 4-position of aromatic rings were favorable for the antibacterial activity against Xoo.

The control effect of compound $\mathbf{3 j}$ against rice bacterial leaf blight was determined by leaf-cutting method. As shown in Table 4 and Fig. 4, the effective curative activity against rice bacterial leaf blight was $40 \%$, which was better than BT (35\%) and TC (30\%). Furthermore, 3j demonstrated better protection activity (47\%) against rice bacterial leaf blight than BT (39\%) and TC (27\%). These results suggest that $3 \mathbf{j}$ effectively inhibit the growth of rice bacterial leaf blight under greenhouse conditions.

\section{Scanning electron microscopy (SEM) studies}

Through the analysis of antibacterial activity, the mechanism of $\mathbf{3 e}$ for $X a c$ and $\mathbf{3 j}$ for Xoo were further studied via SEM. We found that the increase of concentration would lead to the deepening of cell membrane damage, in the control group without treatment with compound, the cell membrane were full and remains intact (Figs. 5A and 6D). Part of the cell membranes began to be destroyed when the concentration was $50 \mu \mathrm{g} / \mathrm{cm}^{3}$ (Figs. 5B and 6E). And most of the cell membrane was destroyed when the concentration was increased to $100 \mu \mathrm{g} / \mathrm{cm}^{3}$, and only a few cells were remained unaffected (Figs. 5C and 6F). These results showed that the damage of cell membrane became more and more serious with the increasing of the compound concentration. These SEM images further confirmed that $3 \mathrm{e}$ and $3 \mathrm{j}$ destroyed the bacterial cell membrane and eventually killed the bacteria.

Curative activity

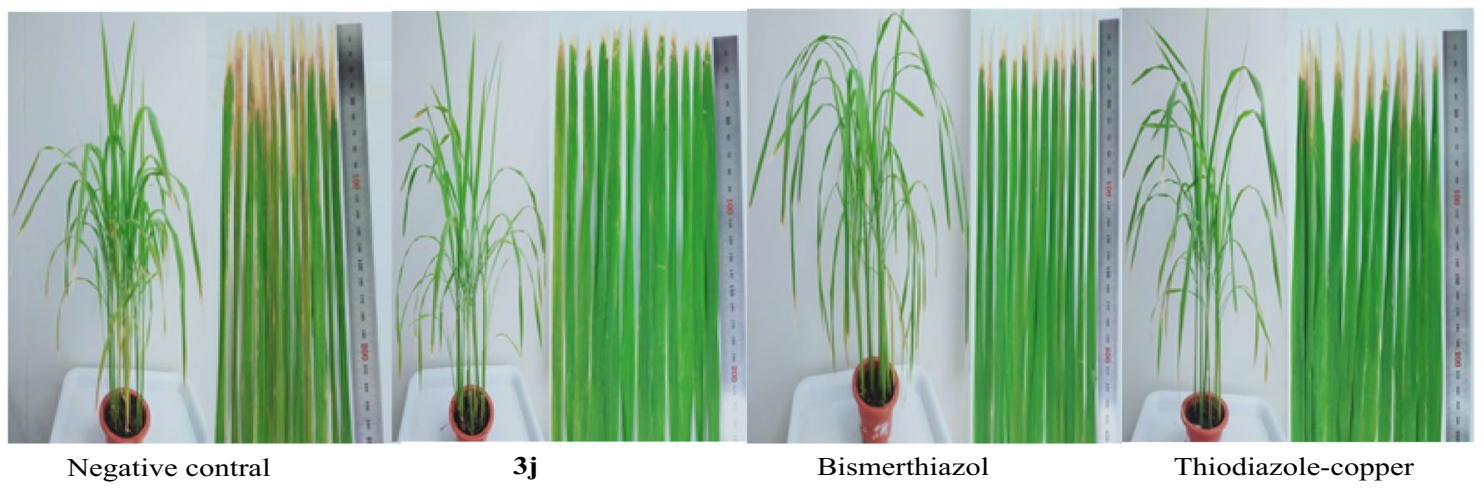

Protection activity

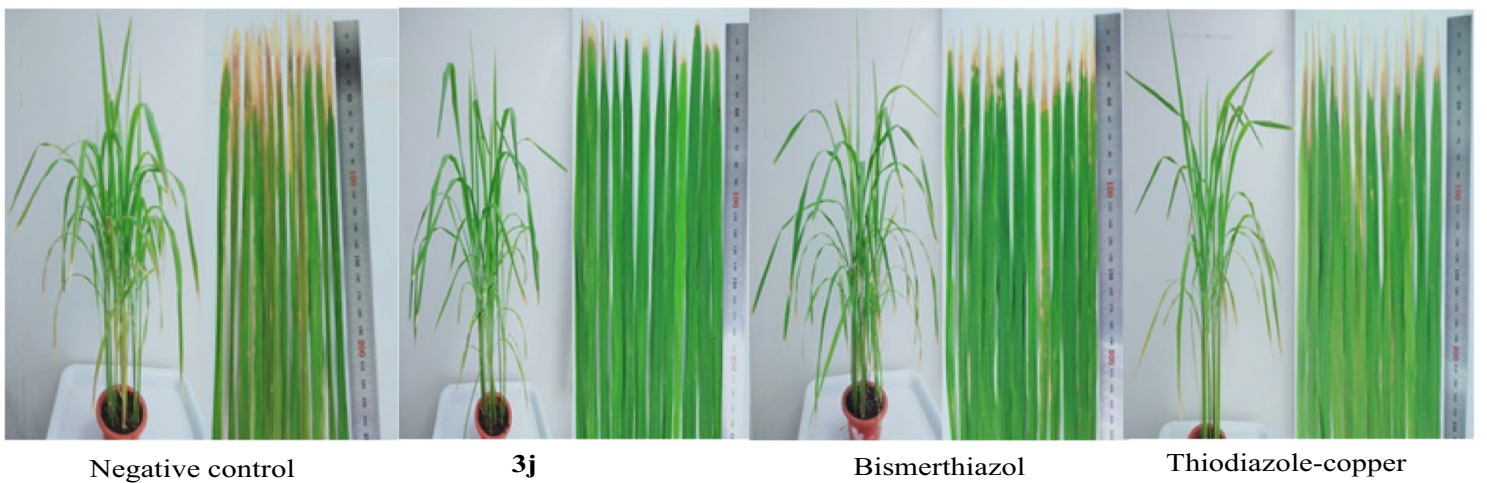

Fig. 4 Curative and protective effects of compound $\mathbf{3 j}\left(200 \mu \mathrm{g} / \mathrm{cm}^{3}\right)$ against Xoo under greenhouse conditions. BT and TC were used as positive controls under similar experimental conditions 
Fig. 5 SEM images for $X a c$ after incubated using different concentrations of compound 3e, a $0 \mu \mathrm{g} / \mathrm{cm}^{3}$, b $50 \mu \mathrm{g} / \mathrm{cm}^{3}$, c $100 \mu \mathrm{g} / \mathrm{cm}^{3}$. Scale bar for $\mathbf{A}-\mathbf{C}$ are $2 \mu \mathrm{m}$
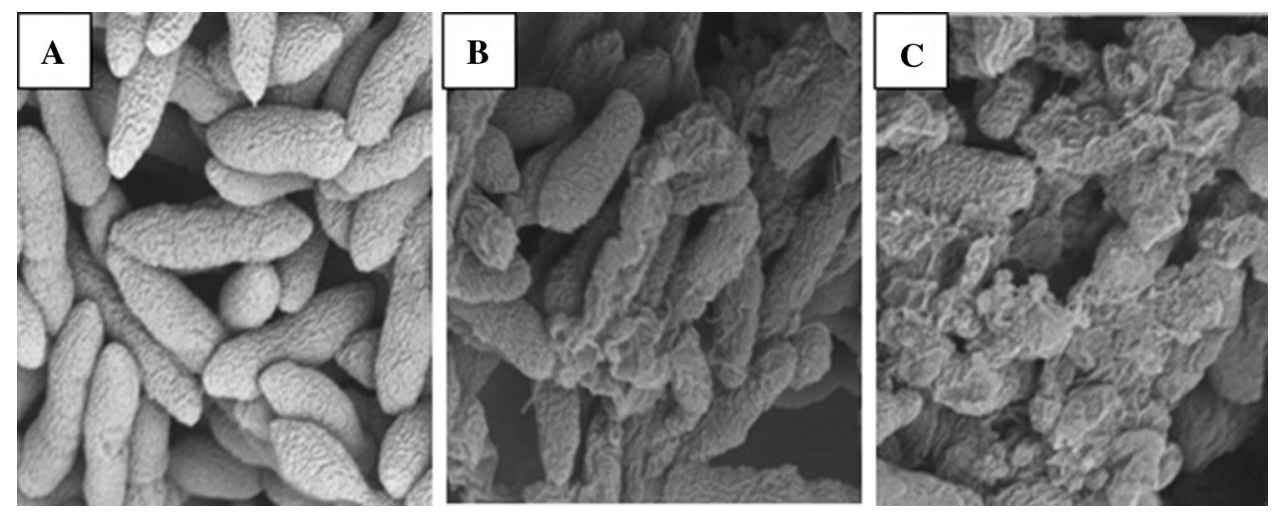

Fig. 6 SEM images for Xoo after incubated using different concentrations of compound $\mathbf{3} \mathbf{j}$, d $0 \mu \mathrm{g} / \mathrm{cm}^{3}$, e $50 \mu \mathrm{g} / \mathrm{cm}^{3}$, and $\mathbf{f}$ $100 \mu \mathrm{g} / \mathrm{cm}^{3}$. Scale bar for D-F are $2 \mu \mathrm{m}$
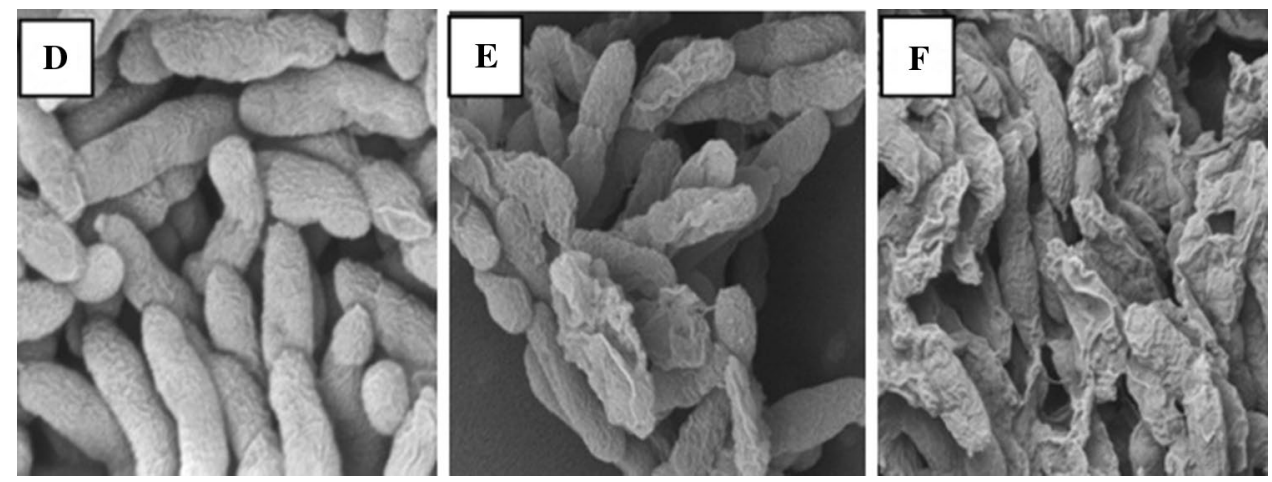

\section{Conclusions}

In conclusion, a series of myricetin derivatives containing sulfonate were designed and synthesized, the antibacterial activity of these derivatives against Xac, Rs, and Xoo have been tested in vitro, and the results indicated that most of compounds have good antibacterial activities. Especially compound $\mathbf{3 e}$ against $\mathrm{Xac}$ and compound $\mathbf{3 j}$ against Xoo wih the $\mathrm{EC}_{50}$ values were $13.76,11.92 \mu \mathrm{g} / \mathrm{cm}^{3}$, respectively, which were superior to BT $\left(50.32\right.$ and $72.08 \mu \mathrm{g} / \mathrm{cm}^{3}$, respectively) and TC ( 83.2 and $99.2 \mu \mathrm{g} / \mathrm{cm}^{3}$, respectively). Compound $\mathbf{3 j}$ also displayed good antibacterial activities against rice bacterial leaf blight (curative activity was $40.7 \%$ and protective activity was $47.9 \%$ ), which were superior to the curative and protection activities of BT (35\% and 39\%) and TC (30\% and 27\%). The SEM images of Xac treated with compound $\mathbf{3 e}$ and $X o o$ treated with compound $\mathbf{3 j}$ revealed that the cell membranes of bacteria are deformed and broken. These results demonstrate that novel myricetin derivatives containing sulfonate could be further studied as new antibacterial compounds.

\section{Experimental}

All reagents and solutions were purchased from Chemical Reagent Company and were analytical grade reagents. The melting point of all synthesized compounds were found by an XT-4 Binocular Microscope melting point apparatus (Beijing Tech. Instrument, China). DMSO- $d_{6}$ were used as a solvent and TMS as an internal standard, a Bruker Ascend-400 spectrometer (Bruker Optics, Switzerland) was used to give the ${ }^{1} \mathrm{H}$ NMR, ${ }^{13} \mathrm{C}$ NMR, and ${ }^{19} \mathrm{~F}$ NMR spectra of title compounds. HRMS data were obtained using Thermo Scientific Q Exactive Hybrid Quadrupole Mass Spectrometer (Thermo Scientific Inc., St Louis, MO, USA). The X-ray crystal data were acquired using a Bruker D8-QUEST diffractometer (Bruker Optics, Switzerland). All ${ }^{1} \mathrm{H}$ NMR, ${ }^{13} \mathrm{C}$ NMR, and ${ }^{19} \mathrm{~F}$ NMR spectra, and HRMS are provided in Supporting Information.

\section{General synthesis procedure for intermediate 1 and intermediate 2}

Based on the previously reported method [36-38], the synthetic route of target compound is listed in Scheme 1. The myricetin, anhydrous potassium carbonate and $N, N$ dimethylformamide (DMF) were added to a round-bottom flask with magnetic stirring; the reaction mixture was allowed to stir for $20 \mathrm{~min}$ at room temperature and potassium iodide was asses dropwise slowly. After completion of the reaction, monitored by TLC plate, the reaction mixture was extracted with dichloromethane and the solvent was removed under reduced pressure to obtain 5,7-dimethoxy3-[(3,4,5-trimethoxy-6-methyltetrahydro- $2 H$-pyran-2-yl)- 
oxy]-2-(3,4,5-trimethoxyphenyl)-4H-chromen-4-one (intermediate 1), which was used for the next step without purification.

Intermediate 1 was added in ethanol and refluxed with stirring for $2 \mathrm{~h}$. Concentrated hydrochloric acid was slowly added dropwise at this temperature, continued to reflux for 2-3 h, and a large amount of solid precipitated when the reaction was cooled to ambient temperature, which was filtered and dried to obtain 3-hydroxy-5,7-dimethoxy-2-(3,4,5trimethoxyphenyl)- $4 H$-chromen-4-one (intermediate 2 ).

\section{General synthesis procedure for target compounds 3a-3v}

Intermediate 2 , anhydrous potassium carbonate and acetonitrile were added to round-bottom flask with magnetic stirring and reflux for $0.5-1 \mathrm{~h}$.

Then substituted benzenesulfonyl chloride was added and continued to reflux for $2-3 \mathrm{~h}$ until completion of the reaction as determined by TLC. The mixture was cooled to room temperature and poured into ice water; the crude products were recrystallized with anhydrous ethanol and DMF to give target compounds $\mathbf{3 a} \mathbf{a}-\mathbf{3 v}$.

5,7-Dimethoxy-4-oxo-2-(3,4,5-trimethoxyphenyl)-4Hchromen-3-yl benzenesulfonate $\left(3 \mathrm{a}, \mathrm{C}_{26} \mathrm{H}_{24} \mathrm{O}_{10} \mathrm{~S}\right)$ Yellow solid; yield: $89 \%$; m.p.: $194-195{ }^{\circ} \mathrm{C}$; ${ }^{1} \mathrm{H}$ NMR $(400 \mathrm{MHz}$, DMSO- $\left.d_{6}\right): \delta=7.71(\mathrm{dd}, J=8.4,1.1 \mathrm{~Hz}, 2 \mathrm{H}, \mathrm{Ph}-\mathrm{H}), 7.64(\mathrm{t}$, $J=7.5 \mathrm{~Hz}, 1 \mathrm{H}, \mathrm{Ph}-\mathrm{H}), 7.45$ (dd, $J=8.2,7.6 \mathrm{~Hz}, 2 \mathrm{H}, \mathrm{Ph}-\mathrm{H}$ ), $6.95(\mathrm{~s}, 2 \mathrm{H}, \mathrm{Ph}-\mathrm{H}), 6.85$ (d, $J=2.2 \mathrm{~Hz}, 1 \mathrm{H}, \mathrm{Ph}-\mathrm{H}), 6.58$ (d, $J=2.2 \mathrm{~Hz}, 1 \mathrm{H}, \mathrm{Ph}-\mathrm{H}$ ), 3.91 (s, $3 \mathrm{H}, \mathrm{Ph}-\mathrm{OCH}_{3}$ ), 3.87 (s, $\left.3 \mathrm{H}, \mathrm{Ph}-\mathrm{OCH}_{3}\right), 3.75$ (s, $\left.6 \mathrm{H}, 2 \times \mathrm{Ph}-\mathrm{OCH}_{3}\right), 3.72$ (s, $3 \mathrm{H}, \mathrm{Ph}-$ $\left.\mathrm{OCH}_{3}\right) \mathrm{ppm} ;{ }^{13} \mathrm{C}$ NMR $\left(101 \mathrm{MHz}\right.$, DMSO- $\left.d_{6}\right): \delta=170.08$, $164.84,160.87,158.69,155.58,152.96,140.18,137.13$, $134.83,133.22,129.58,128.89,128.11,127.97,125.95$, 124.22, 108.34, 106.63, 97.11, 93.99, 60.41, 56.77, 56.68, 56.42 ppm; HRMS (ESI): $\mathrm{m} / z$ calcd. $[\mathrm{M}+\mathrm{H}]^{+} 529.114$, found 529.116 .

5,7-Dimethoxy-4-oxo-2-(3,4,5-trimethoxyphenyl)-4H-chromen-3-yl 4-methylbenzenesulfonate $\left(3 \mathrm{~b}, \mathrm{C}_{27} \mathrm{H}_{26} \mathrm{O}_{10} \mathrm{~S}\right)$ White solid; yield: $81 \%$; m.p.: $170-171{ }^{\circ} \mathrm{C} ;{ }^{1} \mathrm{H}$ NMR $(400 \mathrm{MHz}$, DMSO $\left.-d_{6}\right): \delta=7.56(\mathrm{~d}, J=8.3 \mathrm{~Hz}, 2 \mathrm{H}, \mathrm{Ph}-\mathrm{H}), 7.23$ (d, $J=8.1 \mathrm{~Hz}, 2 \mathrm{H}, \mathrm{Ph}-\mathrm{H}), 6.92$ (s, 2H, Ph-H), 6.83 (d, $J=2.2 \mathrm{~Hz}, 1 \mathrm{H}, \mathrm{Ph}-\mathrm{H}), 6.57(\mathrm{~d}, J=2.2 \mathrm{~Hz}, 1 \mathrm{H}, \mathrm{Ph}-\mathrm{H}), 3.91$ $\left(\mathrm{s}, 3 \mathrm{H}, \mathrm{Ph}-\mathrm{OCH}_{3}\right), 3.87\left(\mathrm{~s}, 3 \mathrm{H}, \mathrm{Ph}-\mathrm{OCH}_{3}\right), 3.76(\mathrm{~s}, 6 \mathrm{H}$, $\left.2 \times \mathrm{Ph}-\mathrm{OCH}_{3}\right), 3.73\left(\mathrm{~s}, 3 \mathrm{H}, \mathrm{Ph}-\mathrm{OCH}_{3}\right), 2.34\left(\mathrm{~s}, 3 \mathrm{H}, \mathrm{Ph}-\mathrm{CH}_{3}\right)$ ppm; ${ }^{13} \mathrm{C}$ NMR (101 MHz, DMSO- $\left.d_{6}\right): \delta=170.12,164.80$, $160.86,158.67,155.58,152.95,145.45,140.29,133.91$, $133.07,130.03,128.10,124.27,108.40,106.62,97.08$, 93.96, 60.55, 56.75, 56.65, 56.40, 21.49 ppm; HRMS (ESI): $\mathrm{m} / z$ calcd. $[\mathrm{M}+\mathrm{H}]^{+}$543.1306, found 543.1319.
5,7-Dimethoxy-4-oxo-2-(3,4,5-trimethoxyphenyl)-4H-chromen-3-yl 4-methoxybenzenesulfonate $(3 \mathrm{c}$, $\mathrm{C}_{27} \mathrm{H}_{26} \mathrm{O}_{11}$ S) White solid; yield: $73 \%$; m.p.: $150-151{ }^{\circ} \mathrm{C} ;{ }^{1} \mathrm{H}$ NMR (400 MHz, DMSO- $\left.d_{6}\right): \delta=7.61-7.57(\mathrm{~m}, 2 \mathrm{H}, \mathrm{Ph}-\mathrm{H})$, $6.91(\mathrm{~s}, 2 \mathrm{H}, \mathrm{Ph}-\mathrm{H}), 6.89$ (d, $J=1.9 \mathrm{~Hz}, 2 \mathrm{H}, \mathrm{Ph}-\mathrm{H}), 6.84$ (d, $J=2.2 \mathrm{~Hz}, 1 \mathrm{H}, \mathrm{Ph}-\mathrm{H}), 6.58(\mathrm{~d}, J=2.2 \mathrm{~Hz}, 1 \mathrm{H}, \mathrm{Ph}-\mathrm{H}), 3.91$ (s, 3H, Ph- $\mathrm{OCH}_{3}$ ), 3.87 (s, 3H, Ph- $\left.\mathrm{OCH}_{3}\right), 3.81$ (s, 3H, Ph$\left.\mathrm{OCH}_{3}\right), 3.77\left(\mathrm{~s}, 6 \mathrm{H}, 2 \times \mathrm{Ph}-\mathrm{OCH}_{3}\right), 3.72\left(\mathrm{~s}, 3 \mathrm{H}, \mathrm{Ph}-\mathrm{OCH}_{3}\right)$ ppm; ${ }^{13} \mathrm{C}$ NMR (101 MHz, DMSO- $\left.d_{6}\right): \delta=170.20,164.78$, $163.93,160.87,158.68,155.58,152.94,140.16,133.00$, 130.52, 127.92, 124.35, 114.79, 108.46, 106.60, 97.09, 93.97, 60.45, 56.77, 56.66, 56.41, 56.20 ppm; HRMS (ESI): $\mathrm{m} / \mathrm{z}$ calcd. $[\mathrm{M}+\mathrm{H}]^{+}$559.1256, found 559.1268.

5,7-Dimethoxy-4-oxo-2-(3,4,5-trimethoxyphenyl)-4H-chromen-3-yl 2-nitrobenzenesulfonate $\left(3 \mathrm{~d}, \mathrm{C}_{26} \mathrm{H}_{23} \mathrm{NO}_{12} \mathrm{~S}\right)$ Yellow solid; yield: 74\%; m.p.: $239-241{ }^{\circ} \mathrm{C}$; ${ }^{1} \mathrm{H}$ NMR $(400 \mathrm{MHz}$, DMSO- $\left.d_{6}\right): \delta=8.06(\mathrm{~d}, J=8.1 \mathrm{~Hz}, 1 \mathrm{H}, \mathrm{Ph}-\mathrm{H}), 7.92(\mathrm{dd}$, $J=12.0,4.4 \mathrm{~Hz}, 2 \mathrm{H}, \mathrm{Ph}-\mathrm{H}), 7.76-7.70(\mathrm{~m}, 1 \mathrm{H}, \mathrm{Ph}-\mathrm{H})$, 7.06 (s, 2H, Ph-H), 6.88 (d, $J=1.9 \mathrm{~Hz}, 1 \mathrm{H}, \mathrm{Ph}-\mathrm{H}), 6.59$ $(\mathrm{d}, J=2.1 \mathrm{~Hz}, 1 \mathrm{H}, \mathrm{Ph}-\mathrm{H}), 3.92$ (s, $\left.3 \mathrm{H}, \mathrm{Ph}-\mathrm{OCH}_{3}\right), 3.86$ (s, 3H, Ph- $\left.\mathrm{OCH}_{3}\right), 3.80\left(\mathrm{~s}, 6 \mathrm{H}, 2 \times \mathrm{Ph}-\mathrm{OCH}_{3}\right), 3.69$ (s, $\left.3 \mathrm{H}, \mathrm{Ph}-\mathrm{OCH}_{3}\right)$ ppm; ${ }^{13} \mathrm{C}$ NMR $\left(101 \mathrm{MHz}\right.$, DMSO- $d_{6}$ ): $\delta=169.77,165.00,160.91,158.80,155.85,153.00,147.49$, $140.25,136.58,133.60,133.28,131.36,129.64,125.14$, $123.86,108.21,106.68,97.23,94.07,60.43,56.82,56.72$, 56.40 ppm; HRMS (ESI): $\mathrm{m} / z$ calcd. $[\mathrm{M}+\mathrm{H}]^{+}$574.0999, found 574.1013 .

5,7-Dimethoxy-4-oxo-2-(3,4,5-trimethoxyphenyl)-4H-chromen-3-yl 4-nitrobenzenesulfonate $\left(3 e, \mathrm{C}_{26} \mathrm{H}_{23} \mathrm{NO}_{12} \mathrm{~S}\right)$ Yellow solid; yield: $71 \%$; m.p.: $220-221{ }^{\circ} \mathrm{C} ;{ }^{1} \mathrm{H}$ NMR $(400 \mathrm{MHz}$, DMSO- $\left.d_{6}\right): \delta=8.27-8.23(\mathrm{~m}, 2 \mathrm{H}, \mathrm{Ph}-\mathrm{H}), 8.03-7.99$ $(\mathrm{m}, 2 \mathrm{H}, \mathrm{Ph}-\mathrm{H}), 6.93(\mathrm{~s}, 2 \mathrm{H}, \mathrm{Ph}-\mathrm{H}), 6.86(\mathrm{~d}, J=2.2 \mathrm{~Hz}$, $1 \mathrm{H}, \mathrm{Ph}-\mathrm{H}), 6.59$ (d, J=2.2 Hz, 1H, Ph-H), 3.91 (s, 3H, $\left.\mathrm{Ph}-\mathrm{OCH}_{3}\right), 3.86\left(\mathrm{~s}, 3 \mathrm{H}, \mathrm{Ph}-\mathrm{OCH}_{3}\right), 3.77$ (s, 6H, $2 \times \mathrm{Ph}-$ $\left.\mathrm{OCH}_{3}\right), 3.67$ (s, $\left.3 \mathrm{H}, \mathrm{Ph}-\mathrm{OCH}_{3}\right) \mathrm{ppm} ;{ }^{13} \mathrm{C} \mathrm{NMR}(101 \mathrm{MHz}$, DMSO- $\left.d_{6}\right): \delta=169.82,164.97,160.88,158.75,155.82$, $152.99,150.60,142.21,140.32,133.15,129.88,124.73$, 123.91, 108.28, 106.69, 97.21, 94.05, 60.37, 56.80, 56.71, 56.49 ppm; HRMS (ESI): $\mathrm{m} / z$ calcd. $[\mathrm{M}+\mathrm{H}]^{+}$574.0999, found 574.1013 .

5,7-Dimethoxy-4-oxo-2-(3,4,5-trimethoxyphenyl)-4H-chromen-3-yl 2-fluorobenzenesulfonate $\left(3 \mathrm{f}, \mathrm{C}_{26} \mathrm{H}_{23} \mathrm{FO}_{10} \mathrm{~S}\right)$ White solid; yield: 83\%; m.p.: $196-198{ }^{\circ} \mathrm{C} ;{ }^{1} \mathrm{H}$ NMR $(400 \mathrm{MHz}$, DMSO- $\left.d_{6}\right): \delta=7.74-7.65(\mathrm{~m}, 2 \mathrm{H}, \mathrm{Ph}-\mathrm{H}), 7.33-7.26(\mathrm{~m}, 2 \mathrm{H}$, $\mathrm{Ph}-\mathrm{H}), 6.98$ (s, 2H, Ph-H), 6.83 (d, $J=2.2 \mathrm{~Hz}, 1 \mathrm{H}, \mathrm{Ph}-\mathrm{H})$, $6.57(\mathrm{~d}, J=2.2 \mathrm{~Hz}, 1 \mathrm{H}, \mathrm{Ph}-\mathrm{H}), 3.90$ (s, $\left.3 \mathrm{H}, \mathrm{Ph}-\mathrm{OCH}_{3}\right), 3.85$ $\left(\mathrm{s}, 3 \mathrm{H}, \mathrm{Ph}-\mathrm{OCH}_{3}\right), 3.79$ (s, $\left.6 \mathrm{H}, 2 \times \mathrm{Ph}-\mathrm{OCH}_{3}\right), 3.73(\mathrm{~s}, 3 \mathrm{H}$, $\mathrm{Ph}-\mathrm{OCH}_{3}$ ) ppm; ${ }^{13} \mathrm{C}$ NMR (101 MHz, DMSO): $\delta=169.82$, $164.89,160.86,158.69,155.78,153.02,140.13,137.78$ $(\mathrm{d}, J=8.7 \mathrm{~Hz}), 133.21,130.41,125.26(\mathrm{~d}, J=13.9 \mathrm{~Hz})$, 
124.12, 117.85, 117.74 (d, $J=20.4 \mathrm{~Hz}), 108.29,106.39$, 97.14, 94.00, 60.41, 56.71, $56.39 \mathrm{ppm} ;{ }^{19} \mathrm{~F}$ NMR (376 MHz, DMSO- $d_{6}$ ): $\delta=-107.54 \mathrm{ppm}$; HRMS (ESI): $\mathrm{m} / z$ calcd. $[\mathrm{M}+\mathrm{H}]^{+}$547.1056, found 547.1068.

5,7-Dimethoxy-4-oxo-2-(3,4,5-trimethoxyphenyl)-4H-chromen-3-yl 4-fluorobenzenesulfonate $\left(3 \mathrm{~g}, \mathrm{C}_{26} \mathrm{H}_{23} \mathrm{FO}_{10} \mathrm{~S}\right)$ White solid; yield: $72 \%$; m.p.: $195-197{ }^{\circ} \mathrm{C}$; ${ }^{1} \mathrm{H}$ NMR $(400 \mathrm{MHz}$, DMSO- $\left.d_{6}\right): \delta=7.80(\mathrm{dd}, J=8.9,5.0 \mathrm{~Hz}, 2 \mathrm{H}, \mathrm{Ph}-\mathrm{H}), 7.27$ $(\mathrm{t}, J=8.8 \mathrm{~Hz}, 2 \mathrm{H}, \mathrm{Ph}-\mathrm{H}), 6.94(\mathrm{~s}, 2 \mathrm{H}, \mathrm{Ph}-\mathrm{H}), 6.85$ (d, $J=2.2 \mathrm{~Hz}, 1 \mathrm{H}, \mathrm{Ph}-\mathrm{H}), 6.59(\mathrm{~d}, J=2.2 \mathrm{~Hz}, 1 \mathrm{H}, \mathrm{Ph}-\mathrm{H})$, $3.91\left(\mathrm{~s}, 3 \mathrm{H}, \mathrm{Ph}-\mathrm{OCH}_{3}\right), 3.87$ (s, $\left.3 \mathrm{H}, \mathrm{Ph}-\mathrm{OCH}_{3}\right), 3.78$ (s, $\left.6 \mathrm{H}, 2 \times \mathrm{Ph}-\mathrm{OCH}_{3}\right), 3.72\left(\mathrm{~s}, 3 \mathrm{H}, \mathrm{Ph}-\mathrm{OCH}_{3}\right) \mathrm{ppm} ;{ }^{13} \mathrm{C} \mathrm{NMR}$ $\left(101 \mathrm{MHz}, \mathrm{DMSO}-d_{6}\right): \delta=170.06,164.87,160.88,158.72$, $155.62,152.99,140.22,133.21(\mathrm{~d}, J=15.4 \mathrm{~Hz}), 131.42$ (d, $J=10.1 \mathrm{~Hz}), 124.21,117.03,116.81,108.36,106.64$, 97.13, 94.00, 60.42, 56.73, 56.46 ppm; ${ }^{19}$ F NMR $(376 \mathrm{MHz}$, DMSO- $d_{6}$ ): $\delta=-103.47 \mathrm{ppm}$; HRMS (ESI): $\mathrm{m} / z$ calcd. $[\mathrm{M}+\mathrm{H}]^{+}$547.1057, found 547.1068.

5,7-Dimethoxy-4-oxo-2-(3,4,5-trimethoxyphenyl)-4H-chromen-3-yl 3-fluorobenzenesulfonate $\left(3 \mathrm{~h}, \mathrm{C}_{26} \mathrm{H}_{23} \mathrm{FO}_{10} \mathrm{~S}\right)$ Yellow solid; yield: $68 \%$; m.p.: $195-196{ }^{\circ} \mathrm{C} ;{ }^{1} \mathrm{H}$ NMR $\left(400 \mathrm{MHz}, \mathrm{DMSO}-d_{6}\right): \delta=7.64-7.59(\mathrm{~m}, 1 \mathrm{H}, \mathrm{Ph}-\mathrm{H}), 7.55-$ 7.49 (m, 3H, Ph-H), 6.95 (s, 2H, Ph-H), 6.85 (d, J=2.2 Hz, $1 \mathrm{H}, \mathrm{Ph}-\mathrm{H}), 6.58$ (d, J=2.2 Hz, 1H, Ph-H), 3.91 (s, 3H, Ph$\mathrm{OCH}_{3}$ ), 3.87 (s, $3 \mathrm{H}, \mathrm{Ph}-\mathrm{OCH}_{3}$ ), 3.77 (s, $6 \mathrm{H}, 2 \times \mathrm{Ph}-\mathrm{OCH}_{3}$ ), 3.72 (s, $\left.3 \mathrm{H}, \mathrm{Ph}-\mathrm{OCH}_{3}\right) \mathrm{ppm} ;{ }^{13} \mathrm{C}$ NMR $(101 \mathrm{MHz}$, DMSO$\left.d_{6}\right): \delta=170.03,164.88,162.99,160.88,160.52,158.74$, 155.61, 152.97, 140.14, 139.13 (d, $J=7.5 \mathrm{~Hz}), 133.27$, $131.97(\mathrm{~d}, J=8.1 \mathrm{~Hz}), 124.22(\mathrm{~d}, J=18.1 \mathrm{~Hz}), 122.29$, 122.08, 115.18, 114.93, 108.32, 106.59, 97.13, 94.01, $60.29,56.73,56.41 \mathrm{ppm} ;{ }^{19} \mathrm{~F}$ NMR (376 MHz, DMSO$\left.d_{6}\right): \delta=-109.88 \mathrm{ppm}$; HRMS (ESI): $m / z$ calcd. $[\mathrm{M}+\mathrm{H}]^{+}$ 547.1051, found 547.1068.

5,7-Dimethoxy-4-oxo-2-(3,4,5-trimethoxyphenyl)-4H-chromen-3-yl 2-chlorobenzenesulfonate $\left(3 \mathrm{i}, \mathrm{C}_{26} \mathrm{H}_{23} \mathrm{ClO}_{10} \mathrm{~S}\right)$ White solid; yield: 65\%; m.p.: $227-229{ }^{\circ} \mathrm{C}$; ${ }^{1} \mathrm{H}$ NMR $(400 \mathrm{MHz}$, DMSO- $\left.d_{6}\right): \delta=7.83$ (dd, $\left.J=7.4,2.1 \mathrm{~Hz}, 1 \mathrm{H}, \mathrm{Ph}-\mathrm{H}\right), 7.77$ (dd, $J=7.5,1.5 \mathrm{~Hz}, 1 \mathrm{H}, \mathrm{Ph}-\mathrm{H}), 7.57-7.47$ (m, 2H, Ph-H), 7.01 (s, 2H, Ph-H), 6.83 (d, J=2.1 Hz, 1H, Ph-H), 6.57 (d, $J=2.1 \mathrm{~Hz}, 1 \mathrm{H}, \mathrm{Ph}-\mathrm{H}), 3.90$ (s, $\left.3 \mathrm{H}, \mathrm{Ph}-\mathrm{OCH}_{3}\right), 3.84$ (s, $\left.3 \mathrm{H}, \mathrm{Ph}-\mathrm{OCH}_{3}\right), 3.82\left(\mathrm{~s}, 6 \mathrm{H}, 2 \times \mathrm{Ph}-\mathrm{OCH}_{3}\right), 3.73$ (s, $\left.3 \mathrm{H}, \mathrm{Ph}-\mathrm{OCH}_{3}\right) \mathrm{ppm} ;{ }^{13} \mathrm{C}$ NMR $\left(101 \mathrm{MHz}\right.$, DMSO- $\left.d_{6}\right)$ : $\delta=169.85,164.92,160.92,158.75,155.93,153.02,140.15$, $137.63,135.84,135.76,133.58,131.39,128.44,124.03$, $120.11,108.31,106.57,97.16,94.04,60.41,56.79,56.68$, 56.49 ppm; HRMS (ESI): $\mathrm{m} / z$ calcd. $[\mathrm{M}+\mathrm{H}]^{+}$563.0762, found 563.0773 .

5,7-Dimethoxy-4-oxo-2-(3,4,5-trimethoxyphenyl)-4H-chromen-3-yl 3-chlorobenzenesulfonate $\left(3 \mathrm{j}, \mathrm{C}_{26} \mathrm{H}_{23} \mathrm{ClO}_{10} \mathrm{~S}\right)$ White solid; yield: 83\%; m.p.: $182-183{ }^{\circ} \mathrm{C}$; ${ }^{1} \mathrm{H}$ NMR $(400 \mathrm{MHz}$, DMSO- $\left.d_{6}\right): \delta=7.84-7.75(\mathrm{~m}, 3 \mathrm{H}, \mathrm{Ph}-\mathrm{H}), 7.41(\mathrm{t}, J=7.9 \mathrm{~Hz}$, $1 \mathrm{H}, \mathrm{Ph}-\mathrm{H}), 6.96(\mathrm{~s}, 2 \mathrm{H}, \mathrm{Ph}-\mathrm{H}), 6.85(\mathrm{~d}, J=2.2 \mathrm{~Hz}, 1 \mathrm{H}$, $\mathrm{Ph}-\mathrm{H}), 6.58$ (d, $J=2.2 \mathrm{~Hz}, 1 \mathrm{H}, \mathrm{Ph}-\mathrm{H}), 3.92$ (s, 3H, Ph$\left.\mathrm{OCH}_{3}\right), 3.88$ (s, $\left.3 \mathrm{H}, \mathrm{Ph}-\mathrm{OCH}_{3}\right), 3.78$ (s, $\left.6 \mathrm{H}, 2 \times \mathrm{Ph}-\mathrm{OCH}_{3}\right)$, $3.73\left(\mathrm{~s}, 3 \mathrm{H}, \mathrm{Ph}-\mathrm{OCH}_{3}\right) \mathrm{ppm} ;{ }^{13} \mathrm{C}$ NMR $(101 \mathrm{MHz}$, DMSO$\left.d_{6}\right): \delta=170.03,164.92,160.92,158.75,155.46,153.02$, $140.20,139.35,137.76,133.30,131.74,130.03,126.94$, 124.08, 122.49, 108.34, 106.51, 97.16, 94.09, 60.22, 56.81, 56.69, 56.44 ppm; HRMS (ESI): $\mathrm{m} / z$ calcd. $[\mathrm{M}+\mathrm{H}]^{+}$ 563.0761, found 563.0773.

5,7-Dimethoxy-4-oxo-2-(3,4,5-trimethoxyphenyl)-4H-chromen-3-yl 4-chlorobenzenesulfonate $\left(3 \mathrm{k}, \mathrm{C}_{26} \mathrm{H}_{23} \mathrm{ClO}_{10} \mathrm{~S}\right)$ Yellow solid; yield: $88 \%$; m.p.: $102-103{ }^{\circ} \mathrm{C} ;{ }^{1} \mathrm{H}$ NMR $(400 \mathrm{MHz}$, DMSO- $\left.d_{6}\right): \delta=7.74-7.69(\mathrm{~m}, 2 \mathrm{H}, \mathrm{Ph}-\mathrm{H}), 7.55-7.49(\mathrm{~m}, 2 \mathrm{H}$, Ph-H), 6.94 (s, 2H, PH-H), 6.84 (d, J=2.1 Hz, 1H, Ph-H), 6.58 (s, 1H, Ph-H), 3.91 (s, 3H, Ph-OCH ${ }_{3}$ ), 3.87 (s, 3H, Ph$\left.\mathrm{OCH}_{3}\right), 3.78\left(\mathrm{~s}, 6 \mathrm{H}, 2 \times \mathrm{Ph}-\mathrm{OCH}_{3}\right), 3.75\left(\mathrm{~s}, 3 \mathrm{H}, \mathrm{Ph}-\mathrm{OCH}_{3}\right)$ ppm; ${ }^{13} \mathrm{C}$ NMR $\left(101 \mathrm{MHz}, \mathrm{DMSO}-d_{6}\right): \delta=169.97,164.87$, $160.87,158.70,155.68,153.04,140.37,139.87,135.55$, $133.05,130.04,129.79,124.10,108.36,106.66,97.13$, 94.01, 60.58, 56.77, 56.67, 56.47 ppm; HRMS (ESI): $\mathrm{m} / \mathrm{z}$ calcd. $[\mathrm{M}+\mathrm{H}]^{+}$563.0764, found 563.0773.

5,7-Dimethoxy-4-oxo-2-(3,4,5-trimethoxyphenyl)-4H-chromen-3-yl 2-bromobenzenesulfonate $\left(3 \mathrm{l}, \mathrm{C}_{26} \mathrm{H}_{23} \mathrm{BrO}_{10} \mathrm{~S}\right)$ White solid; yield: $82 \%$; m.p.: $232-233{ }^{\circ} \mathrm{C} ;{ }^{1} \mathrm{H}$ NMR $(400 \mathrm{MHz}$, DMSO- $\left.d_{6}\right): \delta=7.83(\mathrm{dd}, J=7.4,2.1 \mathrm{~Hz}, 1 \mathrm{H}, \mathrm{Ph}-\mathrm{H}), 7.79$ 7.73 (m, 1H, Ph-H), 7.56-7.47 (m, 2H, Ph-H), 7.00 (s, 2H, $\mathrm{Ph}-\mathrm{H}), 6.83(\mathrm{~d}, J=2.1 \mathrm{~Hz}, 1 \mathrm{H}, \mathrm{Ph}-\mathrm{H}), 6.57(\mathrm{~d}, J=2.1 \mathrm{~Hz}, 1 \mathrm{H}$, $\mathrm{Ph}-\mathrm{H}$ ), 3.90 (s, $3 \mathrm{H}, \mathrm{Ph}-\mathrm{OCH}_{3}$ ), 3.84 (s, 3H, Ph- $\mathrm{OCH}_{3}$ ), 3.82 (s, $\left.6 \mathrm{H}, 2 \times \mathrm{Ph}-\mathrm{OCH}_{3}\right), 3.72\left(\mathrm{~s}, 3 \mathrm{H}, \mathrm{Ph}-\mathrm{OCH}_{3}\right) \mathrm{ppm} ;{ }^{13} \mathrm{C} \mathrm{NMR}$ $\left(101 \mathrm{MHz}, \mathrm{DMSO}-d_{6}\right): \delta=169.87,164.90,160.87,158.74$, 155.93, 152.99, 140.03, 137.56, 135.84, 135.78, 133.55, 131.39, 128.45, 124.02, 120.12, 108.26, 106.46, 97.13, 93.99, 60.40, 56.77, 56.68, 56.44 ppm; HRMS (ESI): $\mathrm{m} / \mathrm{z}$ calcd. $[\mathrm{M}+\mathrm{H}]^{+}$607.0252, found 607.0268.

5,7-Dimethoxy-4-oxo-2-(3,4,5-trimethoxyphenyl)-4H-chromen-3-yl 3-bromobenzenesulfonate $\left(3 \mathrm{~m}, \mathrm{C}_{26} \mathrm{H}_{23} \mathrm{BrO}_{10} \mathrm{~S}\right)$ Yellow solid; yield: $71 \%$; m.p.: $193-194{ }^{\circ} \mathrm{C} ;{ }^{1} \mathrm{H}$ NMR $\left(400 \mathrm{MHz}, \mathrm{DMSO}-d_{6}\right): \delta=7.70(\mathrm{dd}, J=16.1,6.7,1.5 \mathrm{~Hz}$, $2 \mathrm{H}, \mathrm{Ph}-\mathrm{H}$ ), 7.29 (dd, $J=13.2,5.1 \mathrm{~Hz}, 2 \mathrm{H}, \mathrm{Ph}-\mathrm{H}), 6.98$ (s, $2 \mathrm{H}$, $\mathrm{Ph}-\mathrm{H}), 6.83$ (d, $J=2.2 \mathrm{~Hz}, 1 \mathrm{H}, \mathrm{Ph}-\mathrm{H}), 6.57$ (d, $J=2.2 \mathrm{~Hz}$, $1 \mathrm{H}, \mathrm{Ph}-\mathrm{H}$ ), 3.90 (s, 3H, Ph-OCH 3 ), 3.85 (s, 3H, Ph- $\mathrm{OCH}_{3}$ ), $3.79\left(\mathrm{~s}, 6 \mathrm{H}, 2 \times \mathrm{Ph}-\mathrm{OCH}_{3}\right), 3.73\left(\mathrm{~s}, 3 \mathrm{H}, \mathrm{Ph}-\mathrm{OCH}_{3}\right) \mathrm{ppm} ;{ }^{13} \mathrm{C}$ NMR $\left(101 \mathrm{MHz}, \mathrm{DMSO}-d_{6}\right): \delta=169.83,164.90,160.87$, $158.70,155.80,153.02,140.14,137.83,137.74,133.21$, $130.42,125.32,124.12,117.85,117.64,108.30,106.40$, 97.16, 94.02, 60.42, 56.78, 56.67, 56.40 ppm; HRMS (ESI): $\mathrm{m} / z$ calcd. $[\mathrm{M}+\mathrm{H}]^{+}$607.0255, found 607.0268 . 
5,7-Dimethoxy-4-oxo-2-(3,4,5-trimethoxyphenyl)-4H-chromen-3-yl 4-bromobenzenesulfonate (3n, $\left.\mathrm{C}_{26} \mathrm{H}_{23} \mathrm{BrO}_{10} \mathrm{~S}\right)$ White solid; yield: 73\%; m.p.: $131-133{ }^{\circ} \mathrm{C}$; ${ }^{1} \mathrm{H}$ NMR (400 MHz, DMSO- $\left.d_{6}\right): \delta=7.68-7.61(\mathrm{~m}, 4 \mathrm{H}$, Ph-H), 6.95 (s, 2H, Ph-H), 6.85 (d, J=2.2 Hz, 1H, Ph-H), $6.58(\mathrm{~d}, J=2.2 \mathrm{~Hz}, 1 \mathrm{H}, \mathrm{Ph}-\mathrm{H}), 3.91$ (s, 3H, Ph-OCH ${ }_{3}$, 3.87 (s, 3H, Ph-OCH $\left.{ }_{3}\right), 3.79$ (s, 6H, $\left.2 \times \mathrm{Ph}_{-} \mathrm{OCH}_{3}\right), 3.77$ $\left(\mathrm{s}, 3 \mathrm{H}, \mathrm{Ph}-\mathrm{OCH}_{3}\right) \mathrm{ppm} ;{ }^{13} \mathrm{C}$ NMR (101 MHz, DMSO- $\left.d_{6}\right)$ : $\delta=169.96,164.89,160.89,158.70,155.70,153.06,140.44$, $135.93,133.03,132.75,130.04,129.09,124.10,108.37$, 106.71, 97.15, 94.03, 60.66, 56.79, 56.68, 56.51 ppm; HRMS (ESI): $m / z$ calcd. $[\mathrm{M}+\mathrm{H}]^{+} 607.0255$, found 607.0268 .

5,7-Dimethoxy-4-oxo-2-(3,4,5-trimethoxyphenyl)-4Hchromen-3-yl 3-(trifluoromethyl)benzenesulfonate (30, $\left.\mathrm{C}_{27} \mathrm{H}_{23} \mathrm{~F}_{3} \mathrm{O}_{10} \mathrm{~S}\right)$ White solid; yield: $89 \%$; m.p.: $171-172{ }^{\circ} \mathrm{C}$; ${ }^{1} \mathrm{H}$ NMR (400 MHz, DMSO- $\left.d_{6}\right): \delta=8.11(\mathrm{~d}, J=8.1 \mathrm{~Hz}$, 1H, Ph-H), 8.05 (d, J=7.9 Hz, 1H, Ph-H), 7.98 (s, 1H, $\mathrm{Ph}-\mathrm{H}), 7.72$ (t, J=7.9 Hz, 1H, Ph-H), 6.97 (s, 2H, Ph-H), $6.86(\mathrm{~d}, J=2.2 \mathrm{~Hz}, 1 \mathrm{H}, \mathrm{Ph}-\mathrm{H}), 6.59(\mathrm{~d}, J=2.2 \mathrm{~Hz}, 1 \mathrm{H}$, $\mathrm{Ph}-\mathrm{H}), 3.92$ (s, 3H, Ph-OCH $), 3.87$ (s, 3H, Ph-OCH $), 3.76$ $\left(\mathrm{s}, 6 \mathrm{H}, 2 \times \mathrm{Ph}_{-} \mathrm{OCH}_{3}\right), 3.69\left(\mathrm{~s}, 3 \mathrm{H}, \mathrm{Ph}-\mathrm{OCH}_{3}\right) \mathrm{ppm} ;{ }^{13} \mathrm{C}$ NMR (101 MHz, DMSO- $\left.d_{6}\right): \delta=169.99,164.94,160.88$, $158.74,155.45,152.97,140.14,138.72,133.35,132.08$, $131.51(\mathrm{~d}, J=29.7 \mathrm{~Hz}), 130.32,124.32,123.96,108.26$, 106.44, 97.16, 94.04, 60.26, 56.73, $56.35 \mathrm{ppm} ;{ }^{19} \mathrm{~F}$ NMR (376 MHz, DMSO- $d_{6}$ ): $\delta=-61.51 \mathrm{ppm}$; HRMS (ESI): $m / z$ calcd. $[\mathrm{M}+\mathrm{H}]^{+}$597.1029, found 597.1036.

5,7-Dimethoxy-4-oxo-2-(3,4,5-trimethoxyphenyl)-4Hchromen-3-yl 4-(trifluoromethoxy)benzenesulfonate (3p, $\left.\mathrm{C}_{27} \mathrm{H}_{23} \mathrm{~F}_{3} \mathrm{O}_{11} \mathrm{~S}\right)$ White solid; yield: 81\%; m.p.: $189-191{ }^{\circ} \mathrm{C}$; ${ }^{1} \mathrm{H}$ NMR (400 MHz, DMSO- $\left.d_{6}\right): \delta=7.81(\mathrm{dd}, J=7.9$, 1.2 Hz, 1H, Ph-H), 7.66-7.60 (m, 1H, Ph-H), 7.60-7.53 (m, 1H, Ph-H), 7.46 (dd, J=11.0, 4.2 Hz, 1H, Ph-H), 6.99 (s, 2H, $\mathrm{Ph}-\mathrm{H}), 6.82$ (d, J=2.1 Hz, 1H, Ph-H), 6.56 (d, J=2.0 Hz, $1 \mathrm{H}, \mathrm{Ph}-\mathrm{H}$ ), 3.90 (s, 3H, Ph-OCH ${ }_{3}$ ), 3.84 (s, 3H, Ph-OCH ${ }_{3}$, 3.81 (s, 6H, $2 \times \mathrm{Ph}_{-} \mathrm{OCH}_{3}$ ), 3.73 (s, 3H, Ph-OCH${ }_{3}$ ) ppm; ${ }^{13} \mathrm{C}$ NMR (101 MHz, DMSO- $\left.d_{6}\right): \delta=169.84,164.91,160.91$, $158.74,155.94,153.01,140.15,135.92(\mathrm{~d}, J=9.09 \mathrm{~Hz})$, $135.67,133.52,132.36,131.61,131.17,128.03,124.05$, $108.31,106.53,97.16,94.04,60.42,56.73,56.45 \mathrm{ppm} ;{ }^{19} \mathrm{~F}$ NMR (376 MHz, DMSO- $d_{6}$ ): $\delta=-56.74$ ppm; HRMS (ESI): $\mathrm{m} / \mathrm{z}$ calcd. $[\mathrm{M}+\mathrm{H}]^{+}$613.097, found 613.0985 .

5,7-Dimethoxy-4-oxo-2-(3,4,5-trimethoxyphenyl)-4Hchromen-3-yl 4-(tert-butyl)benzenesulfonate (3q, $\left.\mathrm{C}_{30} \mathrm{H}_{32} \mathrm{O}_{10} \mathrm{~S}\right)$ Yellow solid; yield: 70\%; m.p.: $184-185{ }^{\circ} \mathrm{C}$; ${ }^{1} \mathrm{H}$ NMR (400 MHz, DMSO- $\left.d_{6}\right): \delta=7.63(\mathrm{~d}, J=8.6 \mathrm{~Hz}$, 2H, Ph-H), 7.46 (d, J=8.6 Hz, 2H, Ph-H), 7.01 (s, 2H, $\mathrm{Ph}-\mathrm{H}), 6.86$ (d, J=2.2 Hz, 1H, Ph-H), 6.58 (d, $J=2.2 \mathrm{~Hz}$, 1H, Ph-H), 3.92 (s, 3H, Ph-OCH ${ }_{3}$, 3.87 (s, 3H, Ph-OCH ${ }_{3}$, $3.73\left(\mathrm{~s}, 6 \mathrm{H}, 2 \times \mathrm{Ph}_{-} \mathrm{OCH}_{3}\right), 3.72\left(\mathrm{~s}, 3 \mathrm{H}, \mathrm{Ph}-\mathrm{OCH}_{3}\right), 1.27(\mathrm{~s}$, 9H, Ph-C $\left.\left(\mathrm{CH}_{3}\right)_{3}\right)$ ppm; ${ }^{13} \mathrm{C}$ NMR (101 MHz, DMSO- $\left.d_{6}\right)$ : $\delta=170.12,164.83,160.88,158.66,158.07,155.35,152.97$, $140.49,134.12,133.24,128.01,126.43,125.77,124.77$, $124.17,108.35,106.70,97.09,94.01,60.64,56.77,56.66$, 56.43, 35.43, 31.01 ppm; HRMS (ESI): $\mathrm{m} / \mathrm{z}$ calcd. $[\mathrm{M}+\mathrm{H}]^{+}$ 585.1776 , found 585.1788 .

5,7-Dimethoxy-4-oxo-2-(3,4,5-trimethoxyphenyl)-4H-chromen-3-yl 4-cyanobenzenesulfonate $\left(3 \mathrm{r}, \mathrm{C}_{27} \mathrm{H}_{23} \mathrm{NO}_{10} \mathrm{~S}\right)$ Yellow solid; yield: 63\%; m.p.: 237-238 ${ }^{\circ} \mathrm{C}$; ${ }^{1} \mathrm{H}$ NMR $\left(400 \mathrm{MHz}, \mathrm{DMSO}-d_{6}\right): \delta=7.96-7.89(\mathrm{~m}, 4 \mathrm{H}, \mathrm{Ph}-\mathrm{H})$, 6.94 (s, 2H, Ph-H), 6.84 (d, J=2.0 Hz, 1H, Ph-H), 6.57 (d, $J=2.1 \mathrm{~Hz}, 1 \mathrm{H}, \mathrm{Ph}-\mathrm{H}), 3.91$ (s, 3H, Ph-OCH $\left.{ }_{3}\right), 3.87$ (s, $\left.3 \mathrm{H}, \mathrm{Ph}-\mathrm{OCH}_{3}\right), 3.79\left(\mathrm{~s}, 6 \mathrm{H}, 2 \times \mathrm{Ph}-\mathrm{OCH}_{3}\right), 3.76(\mathrm{~s}, 3 \mathrm{H}, \mathrm{Ph}-$ $\left.\mathrm{OCH}_{3}\right) \mathrm{ppm} ;{ }^{13} \mathrm{C}$ NMR (101 MHz, DMSO- $\left.d_{6}\right): \delta=167.74$, $162.83,158.78,156.62,153.63,150.91,138.87,138.24$, $131.49,131.08,126.68,121.85,115.65,114.84,106.18$, 104.60, 95.05, 91.94, 58.40, 54.67, 54.57, 54.41 ppm; HRMS (ESI): $m / z$ calcd. $[\mathrm{M}+\mathrm{H}]^{+} 554.1102$, found 554.1115.

5,7-Dimethoxy-4-oxo-2-(3,4,5-trimethoxyphenyl)-4H-chromen-3-yl thiophene-2-sulfonate ( $3 \mathrm{~s}, \mathrm{C}_{24} \mathrm{H}_{22} \mathrm{O}_{10} \mathrm{~S}_{2}$ ) White solid; yield: $88 \%$; m.p.: $102-103{ }^{\circ} \mathrm{C} ;{ }^{1} \mathrm{H}$ NMR $(400 \mathrm{MHz}$, DMSO- $\left.d_{6}\right): \delta=8.02(\mathrm{dd}, J=5.0,1.3 \mathrm{~Hz}, 1 \mathrm{H}$, thienylH), 7.62 (dd, $J=3.8,1.3 \mathrm{~Hz}, 1 \mathrm{H}$, thienyl-H), 7.04 (dd, $J=4.9,4.0 \mathrm{~Hz}, 1 \mathrm{H}$, thienyl-H), 7.01 (s, 2H, Ph-H), 6.87 (d, $J=2.2 \mathrm{~Hz}, 1 \mathrm{H}, \mathrm{Ph}-\mathrm{H}), 6.59$ (d, J=2.2 Hz, 1H, Ph-H), 3.92 (s, 3H, Ph- $\left.\mathrm{OCH}_{3}\right), 3.88\left(\mathrm{~s}, 3 \mathrm{H}, \mathrm{Ph}-\mathrm{OCH}_{3}\right), 3.80$ (s, $\left.6 \mathrm{H}, 2 \times \mathrm{Ph}_{-} \mathrm{OCH}_{3}\right), 3.74\left(\mathrm{~s}, 3 \mathrm{H}, \mathrm{Ph}-\mathrm{OCH}_{3}\right) \mathrm{ppm} ;{ }^{13} \mathrm{C} \mathrm{NMR}$ $\left(101 \mathrm{MHz}, \mathrm{DMSO}-d_{6}\right): \delta=169.95,164.87,160.89,158.68$, 155.73, 153.04, 140.29, 137.09, 136.19, 135.83, 133.16, 128.43, 124.30, 108.38, 106.66, 97.15, 94.02, 60.49, 56.79, 56.68, 56.50 ppm; HRMS (ESI): $m / z$ calcd. $[\mathrm{M}+\mathrm{H}]^{+}$ 536.0719 , found 536.0727 .

5,7-Dimethoxy-4-oxo-2-(3,4,5-trimethoxyphenyl)-4H-chromen-3-yl pyridine-3-sulfonate (3t, $\mathrm{C}_{25} \mathrm{H}_{23} \mathrm{NO}_{10} \mathrm{~S}$ ) Brown solid; yield: $87 \%$; m.p.: $141-143{ }^{\circ} \mathrm{C}$; ${ }^{1} \mathrm{H}$ NMR $(400 \mathrm{MHz}$, DMSO- $\left.d_{6}\right): \delta=8.84(\mathrm{dd}, J=24.8,3.0 \mathrm{~Hz}, 2 \mathrm{H}, \mathrm{Py}-\mathrm{H}), 8.18$ $(\mathrm{d}, J=8.1 \mathrm{~Hz}, 1 \mathrm{H}, \mathrm{Py}-\mathrm{H}), 7.51$ (dd, $J=8.0,4.8 \mathrm{~Hz}, 1 \mathrm{H}$, Py-H), 7.01 (s, 2H, Ph-H), 6.87 (s, 1H, Ph-H), 6.59 (s, 1H, $\mathrm{Ph}-\mathrm{H}), 3.90$ (d, J=14.5 Hz, 3H, Ph- $\left.\mathrm{OCH}_{3}\right), 3.87$ (s, 3H, $\mathrm{Ph}-\mathrm{OCH}_{3}$ ), 3.77 (d, J=17.6 Hz, 6H, $\left.2 \times \mathrm{Ph}_{-} \mathrm{OCH}_{3}\right), 3.74$ (s, 3H, Ph- $\mathrm{OCH}_{3}$ ) ppm; ${ }^{13} \mathrm{C}$ NMR (101 MHz, DMSO$\left.d_{6}\right): \delta=169.93,164.95,160.90,158.75,155.81,155.09$, $153.08,148.13,140.32,136.09,134.12,133.26,124.53$, 124.01, 108.29, 106.65, 97.20, 94.05, 60.46, 56.81, 56.70, 56.51 ppm; HRMS (ESI): $m / z$ calcd. $[\mathrm{M}+\mathrm{H}]^{+}$530.1099, found 530.1115 . 
5,7-Dimethoxy-4-oxo-2-(3,4,5-trimethoxyphenyl)-4H-chromen-3-yl quinoline-8-sulfonate $\left(3 \mathrm{u}, \mathrm{C}_{29} \mathrm{H}_{253} \mathrm{NO}_{10} \mathrm{~S}\right)$ White solid; yield: $53 \%$; m.p.: $259-260{ }^{\circ} \mathrm{C} ;{ }^{1} \mathrm{H}$ NMR $(400 \mathrm{MHz}$, DMSO- $\left.d_{6}\right): \delta=8.99$ (d, $J=4.2 \mathrm{~Hz}, 1 \mathrm{H}$, quinoline-H), $8.51-$ $8.43(\mathrm{~m}, 1 \mathrm{H}$, quinoline- $\mathrm{H}), 8.25(\mathrm{dd}, J=13.6,6.2 \mathrm{~Hz}, 2 \mathrm{H}$, quinoline-H), 7.66 (dd, $J=7.1,4.4 \mathrm{~Hz}, 2 \mathrm{H}$, quinoline-H), 6.83 (s, 1H, Ph-H), 6.82 (d, J=2.5 Hz, 2H, Ph-H), 6.58$6.53(\mathrm{~m}, 1 \mathrm{H}, \mathrm{Ph}-\mathrm{H}), 3.90\left(\mathrm{~s}, 3 \mathrm{H}, \mathrm{Ph}-\mathrm{OCH}_{3}\right), 3.84(\mathrm{~s}, 3 \mathrm{H}$, $\left.\mathrm{Ph}-\mathrm{OCH}_{3}\right), 3.64\left(\mathrm{~d}, J=4.1 \mathrm{~Hz}, 6 \mathrm{H}, 2 \times \mathrm{Ph}-\mathrm{OCH}_{3}\right), 3.61$ (s, $\left.3 \mathrm{H}, \mathrm{Ph}-\mathrm{OCH}_{3}\right) \mathrm{ppm} ;{ }^{13} \mathrm{C}$ NMR (101 MHz, DMSO- $\left.d_{6}\right)$ : $\delta=170.20,164.78,160.86,158.71,155.49,152.50,152.18$, $143.19,139.61,137.17,135.95,134.71,133.81,132.48$, 128.96, 125.72, 124.20, 123.09, 108.39, 106.28, 97.02, 93.94, 60.30, 56.73, 56.66, 56.18 ppm; HRMS (ESI): $\mathrm{m} / \mathrm{z}$ calcd. $[\mathrm{M}+\mathrm{H}]^{+} 580.1253$, found 580.1271 .

5,7-Dimethoxy-4-oxo-2-(3,4,5-trimethoxyphenyl)-4Hchromen-3-yl 4-acetamidobenzenesulfonate (3v, $\mathrm{C}_{287} \mathrm{H}_{27} \mathrm{NO}_{11} \mathrm{~S}$ ) White solid; yield: $65 \%$; m.p.: $260-261{ }^{\circ} \mathrm{C}$; ${ }^{1} \mathrm{H}$ NMR (400 MHz, DMSO- $d_{6}$ ): $\delta=10.40$ (s, $1 \mathrm{H}, \mathrm{Ph}-\mathrm{NH}-$ ), 7.64-7.57 (m, 4H, Ph-H), 6.93 (s, 2H, Ph-H), 6.84 (d, $J=1.5 \mathrm{~Hz}, 1 \mathrm{H}, \mathrm{Ph}-\mathrm{H}), 6.57$ (d, $J=2.1 \mathrm{~Hz}, 1 \mathrm{H}, \mathrm{Ph}-\mathrm{H}), 3.91$ $\left(\mathrm{s}, 3 \mathrm{H}, \mathrm{Ph}-\mathrm{OCH}_{3}\right), 3.87$ (s, 3H, Ph- $\left.\mathrm{OCH}_{3}\right), 3.76$ (s, 6H, $\left.2 \times \mathrm{Ph}-\mathrm{OCH}_{3}\right), 3.70\left(\mathrm{~s}, 3 \mathrm{H}, \mathrm{Ph}-\mathrm{OCH}_{3}\right), 2.09\left(\mathrm{~s}, 3 \mathrm{H},-\mathrm{CH}_{3}\right)$ ppm; ${ }^{13} \mathrm{C}$ NMR (101 MHz, DMSO- $\left.d_{6}\right): \delta=170.15,169.67$, $164.77,160.86,158.66,155.57,152.98,144.89,140.27$, $132.99,129.58,124.30,118.45,108.45,106.65,97.06$, 93.97, 60.44, 56.74, 56.64, 56.42, 24.64 ppm; HRMS (ESI): $\mathrm{m} / z$ calcd. $[\mathrm{M}+\mathrm{H}]^{+} 586.1369$, found 586.1377 .

\section{Evaluation of the antibacterial activity}

Antibacterial activities of the title compounds against Xanthomonas axonopodispv. citri (Xac), Ralstonia solanacearum (Rs), and Xanthomonas oryzaepv. oryzae (Xoo) were evaluated using the turbidimeter in vitro; commercial agricultural antibacterial bismerthiazol and thiodiazolecopper were used as control. This test method is provided in "Supporting Information".

The protection and curative activities of compound $\mathbf{3 j}$ against rice bacterial leaf blight were determined in potted plants using a complete randomized block design. According to the previously reported method [38], we used $\mathrm{Hu}$ you ming zhan as the experimental seed; this variety is susceptible to rice bacterial blight in recent years. Commercial bactericides BT and TC were used as the positive control samples. This test method is provided in "Supporting Information".

\section{Scanning electron microscope sample preparation}

According to the previously reported method [39], Xac and Xoo were provided with scanning electron microscopy to observe the changes of their cell structure after being treated with different concentrations of drugs. All the bacteria were cultured in NA medium at $28^{\circ} \mathrm{C}$ for $12 \mathrm{~h}$ with active growth. Detailed operating procedures are provided in "Supporting Information".

Supplementary Information The online version contains supplementary material available at https://doi.org/10.1007/s00706-021-02739-1.

Acknowledgements We gratefully acknowledge the Nature Science Foundation of China (No. 21867003), the Science Foundation of Guizhou Province (Nos. 20192452, 20191105, 20171028), Frontiers Science Center for Asymmetric Synthesis and Medicinal Molecules, Department of Education, Guizhou Province (No. 2020004), Program of Introducing Talents of Discipline to Universities of China (111 Program, D20023).

Open Access This article is licensed under a Creative Commons Attribution 4.0 International License, which permits use, sharing, adaptation, distribution and reproduction in any medium or format, as long as you give appropriate credit to the original author(s) and the source, provide a link to the Creative Commons licence, and indicate if changes were made. The images or other third party material in this article are included in the article's Creative Commons licence, unless indicated otherwise in a credit line to the material. If material is not included in the article's Creative Commons licence and your intended use is not permitted by statutory regulation or exceeds the permitted use, you will need to obtain permission directly from the copyright holder. To view a copy of this licence, visit http://creativecommons.org/licenses/by/4.0/.

\section{References}

1. Oliveira AGD, Murate LS, Spago FR, Lopes LD, Beranger JPD, Martin JABS, Nogueira MA, DeMello JCP, Andrade CGT, Andrate G (2011) Biol Control 56:125

2. Li ZF, Wu SL, Bai XF, Liu Y, Lu JF, Liu Y, Xiao BG, Lu XP, Fan L (2011) J Bacteriol 193:6088

3. Lorenzo FD, Palmigiano A, Silipo A, Desaki Y, Garozzo D, Lanzetta R, Shibuya N, Molinaro A (2016) Carbohyd Res 427:38

4. Hu YQ, Zhang S, Xu Z, Lv ZS, Liu CM, Feng LS (2017) Eur J Med Chem 141:335

5. Lin YJ, He ZL, Rosskopf EN, Conn KL, Powell CA, Lazarovits GA (2010) Plant Dis 94:201

6. Perumalsamy S, Bharani M, Sudha M, Nagarajan P, Arul L, Saraswathi R, Balasubramanian P, Ramalingam J (2010) Plant Breed 129:400

7. Zhang SW, Wang RH, Zhao YT, Tareq FS, Sang SM (2019) MolNutr Food Res 63:e1900203

8. Zhang YN, Yin HM, Zhang Y, Zhang DJ, Su X, Kuang HX (2017) J MolStruct 1130:199

9. Wang H, Du YJ, Song HC (2010) J Food Chem 123:6

10. Pasetto S, Pardi V, Murata RM (2014) PLoS ONE 9:1

11. Zhong XM, Wang XB, Chen LJ, Ruan XH, Li Q, Zhang JP, Chen Z, Xue W (2017) Chem Cent J 11:106

12. Wang ZH, Kang KA, Zhang R, Piao MJ, Jo SH, Kim JS (2010) Environ ToxicolPharmacol 29:12

13. Li X, Mai W, Chen D (2014) J Chin ChemSoc 61:383

14. Domitrović R, Rashed K, Cvijanović O, Kneć V, Ević S, Škoda M, Višni A (2015) Chem-Biol Interact 230:21

15. Wang SJ, Tong Y, Lu S, Yang R, Liao X, Xu YF, Li X (2010) Planta Med 76:1492 
16. Xue W, Song BA, Zhao HJ, Qi XB, Huang YJ (2015) Eur J Med Chem 97:155

17. Ha TK, Jung I, Kim ME, Bae SK, Lee JS (2017) Biomed Pharmacother 91:378

18. Liu IM, Zeng TF, Liou SS, Lan TW (2007) Planta Med 73:1054

19. Ma ZG, Wang J, Jiang H, Liu TW, Xie JX (2007) NeuroReport 18:1181

20. Jiang SC, Su SJ, Chen M, Peng F, Zhou Q, Liu TT, Liu LW, Xue W (2020) J Agric Food Chem 68:5641

21. Chen Y, Li P, Su SJ, Chen M, He J, Liu LW, He M, Wang H, Xue W (2019) RSC Adv 9:23045

22. Chen M, Tang XM, Liu TT, Peng F, Zhou Q, Luo H, He M, Xue W (2020) Fitoterapia 149:104804

23. Chen SL, Zhang Y, Liu YX, Wang QM (2019) J Agric Food Chem 67:13

24. Chuang HC, Huang LC, Kapoor M, Liao YJ, Yang CL, Chang CC, Wu CY, Huang TJ, Hu MH (2016) Med ChemComm 7:832

25. Sandra GG, Jesús SM, Eduardo A, Laura D, Angeles FM, Pilar GS, Francesca OM, Rafael G, Javier MF (2011) Eur J InorgChem 10:1657

26. Wahid S, Hanif M, Jahangir S, Shafique M, Shahid H, Muhammad H (2019) J MolStruct 1184:569

27. Tangeti VS, Satyanarayana KVV (2019) Asian J Chem 31:773

28. Guo T, Xia RJ, Chen M, He J, Su SJ, Liu LW, Li XY, Xue W (2019) RSC Adv 9:24942
29. Wang R, Zhi X, Li J, Xu H (2015) J Agric Food Chem 63:6668

30. Sun R, Wang Z, Li Y, Xiong L, Liu Y, Wang Q (2013) J Agric Food Chem 61:517

31. Betts LM, Tam NC, Kabir SMH, Langler RF, Crandal I (2006) Aust J Chem 59:277

32. Peng Y, Shi Y, Zhang H, Mine YT, Sao R (2017) J Funct Food 35:635

33. Kang GQ, Duan WG, Lin GS, Yu YP, Wang XY, Lu SZ (2019) Molecules 24:477

34. Chen L, Wang PY, Li ZX, Zhou L, Wu ZB, Song BA, Yang S (2016) Chin J Chem 34:1236

35. Li P, Hu DY, Xie DD, Chen JX, Jin LH, Song BA (2018) J Agric Food Chem 66:3093

36. Tang X, Zhang C, Chen M, Xue YN, Liu TT, Xue W (2020) New J Chem 44:2374

37. Ruan XH, Zhang C, Jiang SC, Guo T, Xia RJ, Chen Y, Tang X, Xue W (2018) Molecules 23:3132

38. Jiang SC, Tang X, Chen M, He J, Su SJ, Liu LW, He M, Xue W (2020) Pest ManagSci 76:853

39. Tang X, Su SJ, Chen M, He J, Xia RJ, Guo T, Chen Y, Zhang C, Wang J, Xue W (2019) RSC Adv 9:6011

Publisher's Note Springer Nature remains neutral with regard to jurisdictional claims in published maps and institutional affiliations. 